UC-802

Issued: February 1995

Mineralogic Variation in

Drill Core UE-25 UZ\#16,

Yucca Mountain, Nevada
S. J. Chipera
D. T. Vaniman
B. A. Carlos
D. L. Bish 


\section{DISCLAIMER}

This report was prepared as an account of work sponsored by an agency of the United States Government. Neither the United States Government nor any agency thereof, nor any of their employees, make any warranty, express or implied, or assumes any legal liability or responsibility for the accuracy, completeness, or usefulness of any information, apparatus, product, or process disclosed, or represents that its use would not infringe privately owned rights. Reference herein to any specific commercial product, process, or service by trade name, trademark, manufacturer, or otherwise does not necessarily constitute or imply its endorsement, recommendation, or favoring by the United States Government or any agency thereof. The views and opinions of authors expressed herein do not necessarily state or reflect those of the United States Government or any agency thereof. 


\section{DISCLAIMER}

Portions of this document may be illegible in electronic image products. Images are produced from the best available original document. 


\title{
MINERALOGIC VARIATION IN DRILL CORE UE-25 UZ\#16, YUCCA MOUNTAIN, NEVADA
}

\author{
by
}

\author{
S. J. Chipera, D. T. Vaniman, B. A. Carlos, and D. L. Bish
}

\begin{abstract}
Quantitative X-ray powder diffraction methods have been used to analyze 108 samples from drill core UE-25 UZ\#16 at Yucca Mountain, Nevada. This drill hole, located within the imbricate fault zone east of the potential Yucca Mountain repository site, confirms our previous knowledge of gross-scale mineral distributions at Yucca Mountain and provides insight into possible shallow pathways for hydrologic recharge into the potential host rock. Analyses of samples from UE-25 UZ\#16 have shown that the distribution of major zeolitized horizons, of silica phases, and of glassy tuffs are similar to those noted in nearby drill cores. However, the continuous core and closer sample spacing in UE-25 UZ\#16 provide a more exact determination of mineral stratigraphy, particularly in hydrologically important units such as the Paintbrush bedded tuffs above the Topopah Spring Tuff and in the upper vitrophyre of the Topopah Spring Tuff. The discovery of matrix zeolitization in the devitrified Topopah Spring Tuff of UE25 UZ\#16 shows that some unexpected mineralogic features can still be encountered in the exploration of Yucca Mountain and emphasizes the importance of obtaining a more complete three-dimensional model of Yucca Mountain mineralogy.
\end{abstract}

\section{INTRODUCTION}

Yucca Mountain, located in southwestern Nevada, is being investigated to determine its suitability to host the first high-level nuclear waste repository in the United States (Fig. 1). The studies of Yucca Mountain and the surrounding area are part of the Yucca Mountain Site Characterization Project, directed by the Yucca Mountain Site Characterization Office (YMSCO) of the U.S. Department of Energy, Nevada Operations Office. It is important to understand the mineral content and distribution within the mountain to determine how it will perform should a waste repository be emplaced within it. Bish and Chipera (1989) compiled a summary of mineral distribution with depth for drill holes analyzed for Yucca Mountain. Their results, however, were based on data from drill holes that were typically drilled under different 
conditions (e.g., using polymer drilling mud rather than drilling dry), and the holes were not drilled under today's quality assurance (QA) program. Drill hole UE-25 UZ\#16 was one of the first holes drilled under the present QA program, and it provides the first core obtained by dry drilling, using air circulation. Quantitative mineralogical results from this drill hole are compared with findings from previous drill holes to augment our understanding of the mineralogy at Yucca Mountain by providing samples from a new locality (Fig. 1). In addition, sampling was designed to examine every textural/lithologic subunit in order to test whether there is any relation between mineralogy and textural/lithologic features in the rock.

This report provides insight into possible shallow pathways for hydrologic recharge into the potential host rock at Yucca Mountain. This upper interval is particularly important in defining the chemistry and extent of hydrologic recharge into the unsaturated zone. Hydrologic models suggest that the upper $76 \mathrm{~m}$ (250 ft) of tuffs at Yucca Mountain are not in steady-state equilibrium with the surface and are susceptible to broadly varying water flux with variations in climate (Flint et al. 1993). The chemistry of waters entering Yucca Mountain from the surface is largely determined by soil-zone reactions, possibly including dissolution of cristobalite and tridymite (Vaniman et al. 1993), or by reaction with the large amounts of glass that occur in the nonwelded interval between the Tiva Canyon and Topopah Spring Tuffs (Vaniman et al. 1993).

The units represented by the sampled interval in drill hole UE-25 UZ\#16 (preliminary stratigraphy obtained from D. Buesch of the USGS) are as follows:

\section{Depth Stratigraphic Unit}

$\begin{aligned} 0-12.1 \mathrm{~m}: & \text { Alluvium } \\ 12.1-48.98 \mathrm{~m}: & \text { Tiva Canyon Tuff } \\ 48.98-63.92 \mathrm{~m}: & \text { Paintbrush bedded tuffs } \\ 63.92-366.16 \mathrm{~m}: & \text { Topopah Spring Tuff } \\ 366.16-452.63 \mathrm{~m}: & \text { Calico Hills Formation } \\ 452.63-\mathrm{TD} \mathrm{m}: & \text { Prow Pass Tuff }\end{aligned}$

The Paintbrush bedded tuffs, in the upper portion of UE-25 UZ\#16 between the Tiva Canyon and Topopah Spring Tuffs, are of particular interest. Previous studies of this nonwelded interval at Yucca Mountain have shown that it is variably glassy or clay-rich, with apparently sporadic occurrence of zeolites. However, previous studies of the mineralogy in this interval from other drill holes are not sufficiently detailed to determine if textural/lithologic variation (e.g., degree of welding, pumice orientation, abundance of lithophysae, variation in color, etc.) affects alteration mineralogy. The textural/lithologic variations in this interval are very complex; one of the goals of this study was to determine whether these complex variations 


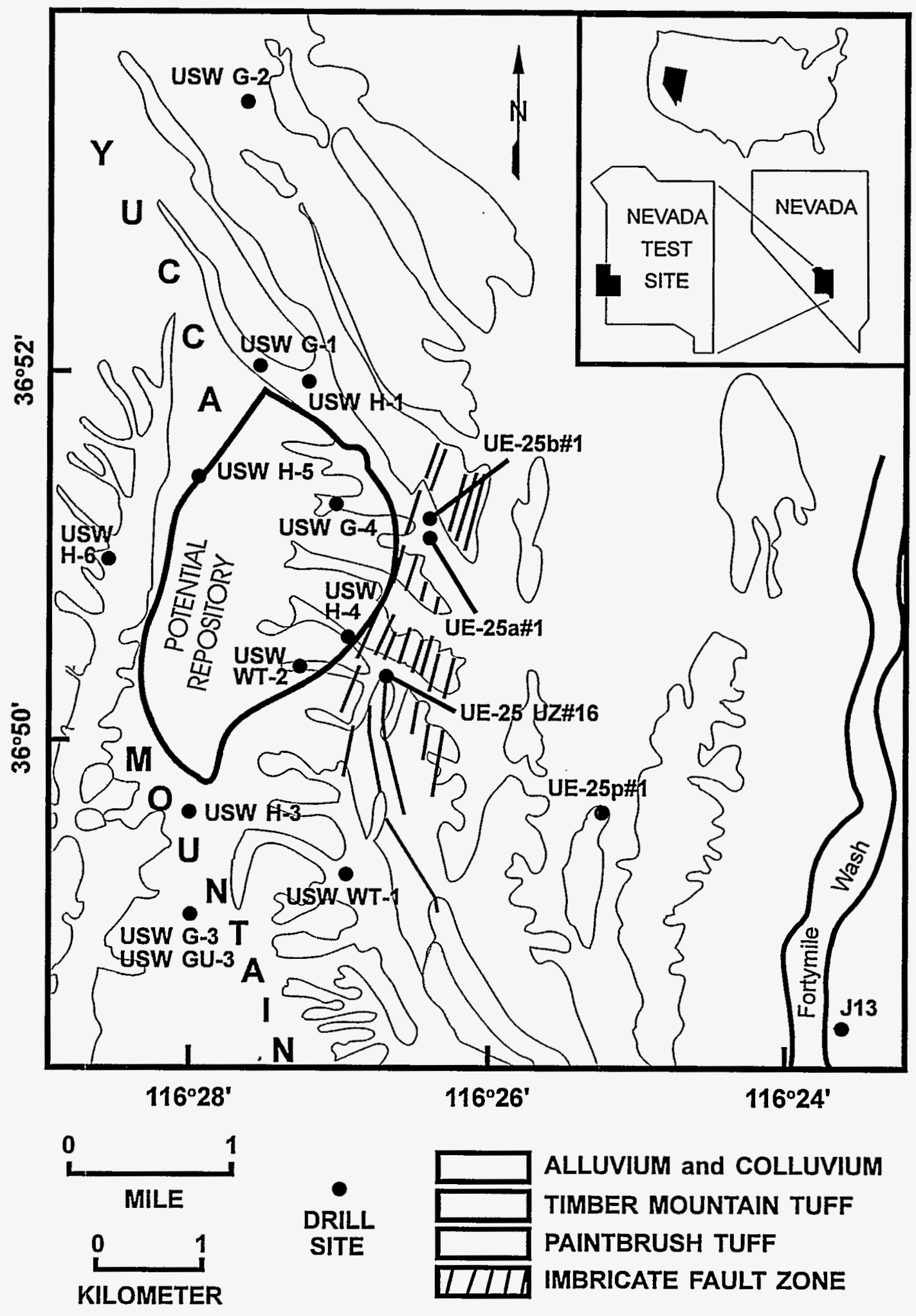

Fig. 1. Map of Yucca Mountain, Nevada, showing the locations of the drill holes mentioned in the text. 
are reflected in an equally complex mineral stratigraphy.

The thin but hydrologically important upper vitrophyre of the Topopah Spring Tuff lies beneath the nonwelded Paintbrush bedded tuffs. This vitrophyre has a significant hydrologic impact despite its meager thickness of $\sim 3 \mathrm{~m}$ because it is the greatest single barrier to downward recharge into the unsaturated zone (Flint et al. 1993). One of the important features examined in this vitrophyre is the extent of alteration, indicating whether this glassy barrier has been compromised by 12 Myr of potential recharge into the unsaturated zone. If the extent of alteration in the upper Topopah vitrophyre is variable across Yucca Mountain, then it may be possible to map those portions of the mountain where recharge has been, and is likely to be, concentrated.

The Topopah Spring Tuff is critical to site characterization because it is the host rock for the potential high-level nuclear waste repository. The potential repository horizon is located in the lower part of the Topopah Spring Tuff, which consists of densely welded, devitrified rhyolitic tuff (Broxton et al. 1989). This rhyolitic tuff is chemically homogeneous, but the mineralogy is variable, particularly in the relative abundances of silica polymorphs (quartz, cristobalite, and tridymite). These silica minerals are important, because the least stable of them (cristobalite and tridymite) can affect the aqueous activity of silica in local unsaturatedzone waters. Moreover, the least stable silica minerals provide a ready source of silica for dissolution, transport, and deposition under the thermal perturbations of a repository. In that part of the Topopah Spring Tuff in which the potential repository might be constructed, cristobalite in particular is an abundant and relatively unstable silica mineral (Bish et al. 1984).

The effects of thermal alteration by hot emplaced waste may be most pronounced in the underlying basal vitrophyre of the Topopah Spring Tuff. This vitrophyre is variably altered across Yucca Mountain, with devitrification-associated heulandite formation penetrating its highly irregular upper surface (Levy and O'Neil 1989). Clinoptilolite and other zeolites (e.g., erionite) may invade the lower margin of the basal vitrophyre.

At the eastern part of Yucca Mountain, the nonwelded base of the Topopah Spring Tuff and all of the underlying Calico Hills Formation is extensively zeolitized (clinoptilolite \pm mordenite); the silica phase associated with this zeolitization is opal-CT. In the western part of Yucca Mountain, the few core samples available suggest that this entire interval is essentially vitric and unaltered. The data suggest an alteration front that may be associated with past rises in the static water level (SWL), but the nature of this zeolitic/vitric transition and the implications for past fluctuations in the SWL will remain speculative until further core samples are obtained from within the potential repository block. The nature of the lateral and vertical transitions between zeolitized and vitric portions of the Calico Hills Formation is of fundamental importance to understanding (1) the transport pathways from the potential repository toward the saturated 
zone and (2) the potential for thermal alteration or dehydration of existing zeolites and possible formation of new zeolites from heated glass (Vaniman and Bish, in press).

The Calico Hills Formation at the potential repository site is underlain by the mostly devitrified, poorly welded Prow Pass Tuff of the Crater Flat Group. The data available to date suggest that cores taken from locations in which the Prow Pass is high above the SWL and underlies vitric portions of the Calico Hills Formation contain the same variable silica-polymorph associations (tridymite, cristobalite, and quartz) found in the devitrified Topopah Spring Tuff (e.g., USW GU-3 in Vaniman et al. 1984). In cores where the Prow Pass is closer to or within the saturated zone and is overlain by zeolitized portions of the Calico Hills Formation, only quartz is preserved (Bish and Chipera 1989). If borne out in further drill cores, this alterationassociated variability in the Prow Pass may prove to be an important component in predicting the future silica activity, and hence the extent of silica mobilization, to be expected in thermallyperturbed portions of the Prow Pass Tuff.

\section{METHODS}

The mineralogy of 108 samples ranging in depth from 12.0 to 513.3 meters (39.2 to $1684.0 \mathrm{ft}$ ) from drill core UE-25 UZ\#16 was quantitatively analyzed by $X$-ray powder diffraction (XRD) methods. Sampling of UE-25 UZ\#16 was performed with a maximum sample spacing of $\sim 6 \mathrm{~m}(20 \mathrm{ft})$ and an average sample spacing of $4.9 \mathrm{~m}(16 \mathrm{ft})$. This sample spacing is much closer than in our previous studies, in which the average spacing between samples was typically $>10 \mathrm{~m}$ (Bish and Chipera 1989). Sample selection in UE-25 UZ\#16 was designed to examine every textural/lithologic subunit in order to test any possible mineralogic significance to subunit distinctions based on appearance (e.g., pumice sizes, pumice abundances, pumice color, core consolidation, matrix color, or apparent clay and zeolite content).

\section{Experimental Techniques}

To prepare the samples, a small portion of each sample $(\sim 0.8 \mathrm{~g})$ was mixed with 1.0 $\mu \mathrm{m}$ corundum $\left(\mathrm{Al}_{2} \mathrm{O}_{3}\right)$ internal standard in the ratio $80 \%$ sample to $20 \%$ corundum by weight. Each sample was then ground under acetone in an automatic Brinkmann Micro-Rapid mill (fitted with an agate mortar and pestle) for a time greater than 10 minutes. This produced a sample with an average particle size of less than $5 \mu \mathrm{m}$ and ensured thorough mixing of sample and internal standard. The fine particle size is necessary to ensure adequate particle statistics and to reduce primary extinction and other sample-related effects (Bish and Reynolds 1989; Klug and Alexander 1974). The adequacy of grinding times and techniques has been confirmed utilizing a Horiba CAPA-500 automatic particle-size-distribution analyzer calibrated with Duke Scientific glass microspheres. 
All diffraction patterns were obtained on a Siemens D500 X-ray powder diffractometer using CuK $\alpha$ radiation, incident- and diffracted-beam Soller slits, and a Kevex Si(Li) solid-state detector from $2-50^{\circ} 2 \theta$, using $0.02^{\circ}$ steps, and counting for at least $2 \mathrm{~s} / \mathrm{step}$. Mineral identification was accomplished by comparing observed patterns with patterns of pure standards, published patterns from the Joint Committee on Powder Diffraction Standards (JCPDS 1986), or calculated mineral patterns obtained from the program POWD10 (Smith et al. 1982).

\section{Quantitative Analysis}

All quantitative analyses for this report employed the internal standard or "matrixflushing" method of Chung (1974) using synthetic 1.0- $\mu \mathrm{m}$ corundum as the internal standard. This method requires that reference intensity ratios (RIRs) be determined before sample analysis. It is important to note that the methods of data collection and analysis used for these samples are not conducive to identifying phases present below $\sim 0.5 \mathrm{wt} \%$, and potentially important trace phases may be present in the samples. Analysis for such trace phases requires either special data collection techniques (e.g., Bish and Chipera 1991) or concentration by magnetic or heavy-liquid methods. The analysis of trace phases is being conducted as a separate activity.

Our methods of quantitative XRD analysis are outlined in Bish and Chipera (1986, 1988, and 1989). The computer program QUANT (version 5.05) was used to reduce the XRD data. This version functions identically to previous versions except for modifications and improvements that are discussed below.

In past versions of QUANT, the RIR values for tridymite were obtained from a tridymite-rich sample from the Nevada Test Site. This sample contained significant feldspar, which required normalization of the tridymite: $\mathrm{Al}_{2} \mathrm{O}_{3}$ standard data to compensate for the presence of feldspar. QUANT (version 5.05) uses RIR values measured on two tridymite standards: USW G-1 619 at Yucca Mountain and sample OU-1106 Strat 2-18 from the Pajarito Plateau, Los Alamos, NM. These tridymite standards were separated from the bulk rock using sodium polytungstate heavy liquid (Torresan 1987), resulting in a standard with only trace feldspar impurities. Current RIR values when compared with the past RIR values (Table 1) show that the RIR value for the $20.6^{\circ}$ peak changed only slightly (old $=1.18$, current $=1.16$ ) although a significant difference in RIR value was obtained for the $21.9^{\circ}$ peak (old $=1.10$, current=2.05). Past analyses, however, are still acceptable since the $20.6^{\circ}$ peak is always used when conducting an analysis for tridymite whereas the $21.9^{\circ}$ peak was only input when cristobalite was not present as a major phase. 
Because stellerite was present in the drill core, QUANT was modified to accept stellerite as a phase for analysis, requiring that appropriate RIR values be determined. Concentrated stellerite was obtained from sample UE-25 UZ\#16 1029 using sodium polytungstate heavy liquid to produce approximately $300 \mathrm{mg}$ of purified stellerite. Since only a small amount of stellerite was obtained (which still contained $\sim 20 \%$ impurities of cristobalite and feldspar and with which stellerite has significant peak overlaps), an indirect method for obtaining RIR values was used. The stellerite sample was examined by XRD and the data were analyzed using Rietveld analysis (Post and Bish 1989) to refine the crystal structure of the stellerite which occurs in UE-25 UZ\#16. The stellerite structure of Galli and Alberti (1975) was used as the starting model for the Rietveld analysis. The refined structure was then input into POWD10 (Smith et al. 1982) to calculate RIR values that were incorporated into QUANT. RIR values for stellerite are listed in Table 1.

TABLE 1

New RIR Values In QUANT Version 5.05

$\begin{array}{ccc}20.6^{\circ} \text { peak } & 1.16 & 1.18 \\ 21.7^{\circ} \text { peak } & 2.05 & 1.10 \\ 23.3^{\circ} \text { peak } & 0.63 & \text { not used }\end{array}$

\begin{tabular}{ll} 
Current & $\begin{array}{c}\text { Previous } \\
\text { Values }\end{array}$ \\
\hline
\end{tabular}

\section{Tridymite}

not used

$9.7^{\circ}$ peak

0.46

not used

\section{Feldspar}

$27.0-28.75^{\circ}$ intensity region not used

$$
\begin{aligned}
& \text { Equation to determine RIR for } \\
& \text { the } 13.4^{\circ} \text { sum peak } \\
& \text { Equation to determine RIR for } \\
& \text { the } 23.6^{\circ} \text { peak } \\
& \mathrm{RIR}_{13.4}=-0.0138+2.3659 /\left(\mathrm{INT}_{13.4} / \mathrm{INT}_{27.0-28.75}\right) \\
& \mathrm{RIR}_{23.6}=0.0223+1.8231 /\left(\mathrm{INT}_{23.6} / \mathrm{INT}_{27.0-28.75}\right)
\end{aligned}
$$

The final modification to QUANT consists of an improved method for the determination of feldspar abundance. Feldspar, one of the more abundant minerals in tuffaceous rocks, has significant chemical and structural variability which makes it also one of the most difficult - minerals to quantify accurately and precisely by XRD. The improved method makes use of the feldspar intensity region from $27.0^{\circ}$ to $28.75^{\circ} 2 \theta$ and calculates more accurate RIR values for the $13.4^{\circ}$ sum peak and the $23.6^{\circ}$ peak from predetermined calibration curves which ratio these 
peaks to the $27.0-28.75^{\circ}$ intensity region as outlined in Moore et al. (1993) and Chipera and Bish (in press). Figures 2 and 3 show the curves from which more accurate RIR values can be obtained for the $13.4^{\circ}$ sum peak and $23.6^{\circ}$ peak, respectively. Experimental results on test samples containing known mineral abundances showed that these curves produced significantly more accurate results for the determination of feldspar abundance, even when the samples contained mixtures of several feldspar species.

\section{RESULTS}

Table 2 and Fig. 4 provide the quantitative XRD results for drill hole UE-25 UZ\#16. Results for the upper portion of the drill core (to $73 \mathrm{~m}$ depth) show that the main rock types in this interval consist of (1) the devitrified Tiva Canyon Tuff (to $49 \mathrm{~m}$ depth) underlain by (2) vitricnonwelded tuff to $67 \mathrm{~m}$ depth, (3) a thin zone ( $\sim 3 \mathrm{~m}$ thick) of zeolite-rich nonwelded devitrified tuff ( $\sim 18 \%$ clinoptilolite/heulandite) to $70 \mathrm{~m}$ depth, and (4) the upper vitrophyre of the Topopah Spring Tuff to $73 \mathrm{~m}$ depth. Quartz and cristobalite are fairly ubiquitous throughout this interval of UE-25 UZ\#16. Tridymite, however, occurs only in the upper devitrified portions of the Tiva Canyon Tuff. Hematite is most common in the devitrified and zeolitized tuffs of this upper interval. Trace amounts of calcite are most common in the lower half of the nonwelded section above the upper vitrophyre of the Topopah Spring Tuff. Smectite, although present in all samples, attains significant abundances (up to $\sim 20 \%$ ) only in the nonwelded vitric tuff. In addition, a fracture at $34.4 \mathrm{~m}(112.9 \mathrm{ft})$ in depth was found to be filled with smectite. This smectite was further analyzed by XRD and was identified as a dioctahedral smectite composed of $\sim 100 \%$ expandable smectite (pure smectite with no illite interstratification). Characterization was conducted by comparing the $X$-ray pattern obtained from an oriented ethylene-glycolsolvated sample mount with patterns calculated using the computer program NEWMOD (Reynolds and Reynolds 1987). The complete absence of illite interstratification suggests that the smectite in the vitric zone formed at low temperature and may be currently forming as a low-temperature alteration product of the glass.

The three-meter-thick zeolitized zone above the upper vitrophyre of the Topopah Spring Tuff is of particular interest because it is of sharply restricted occurrence, suggesting "ponded" alteration above the relatively impermeable upper vitrophyre. These zeolites are of the clinoptilolite/heulandite group, with 020:200 intensity ratios indicative of heulandite. To confirm the identification as heulandite, aliquots from samples 223.7/224.2, 224.1/224.7, and $227.0 / 227.2$ were heated overnight at $400^{\circ} \mathrm{C}$ and $550^{\circ} \mathrm{C}$ and then analyzed after each heating 


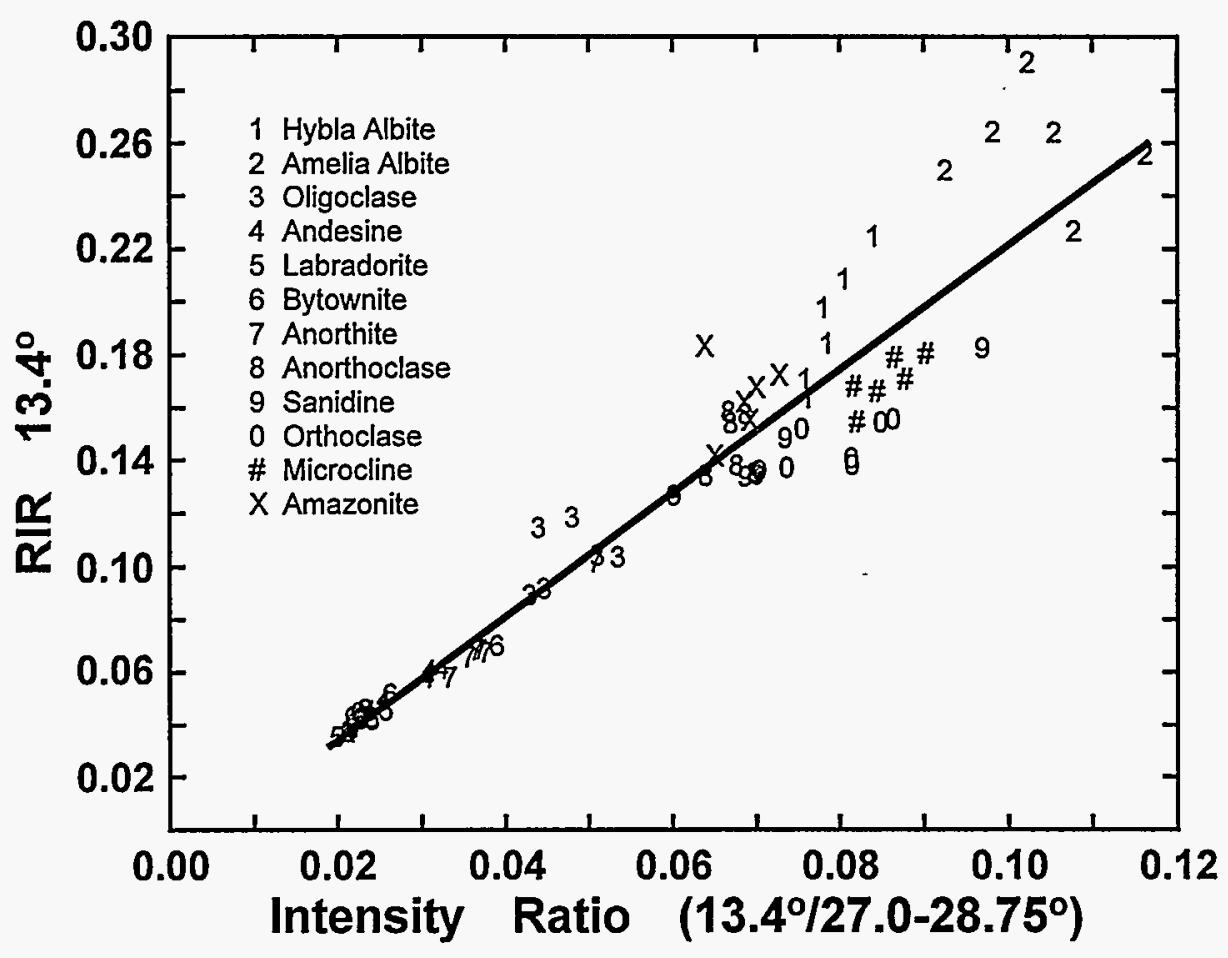

Fig. 2. Relationship between the RIR for the $13.4^{\circ}$ feldspar sum peak and the intensity ratio of the $13.4^{\circ}$ sum peak to the sum of the reflections in the $27.0-28.75^{\circ}$ region (CuK $\alpha$ ).

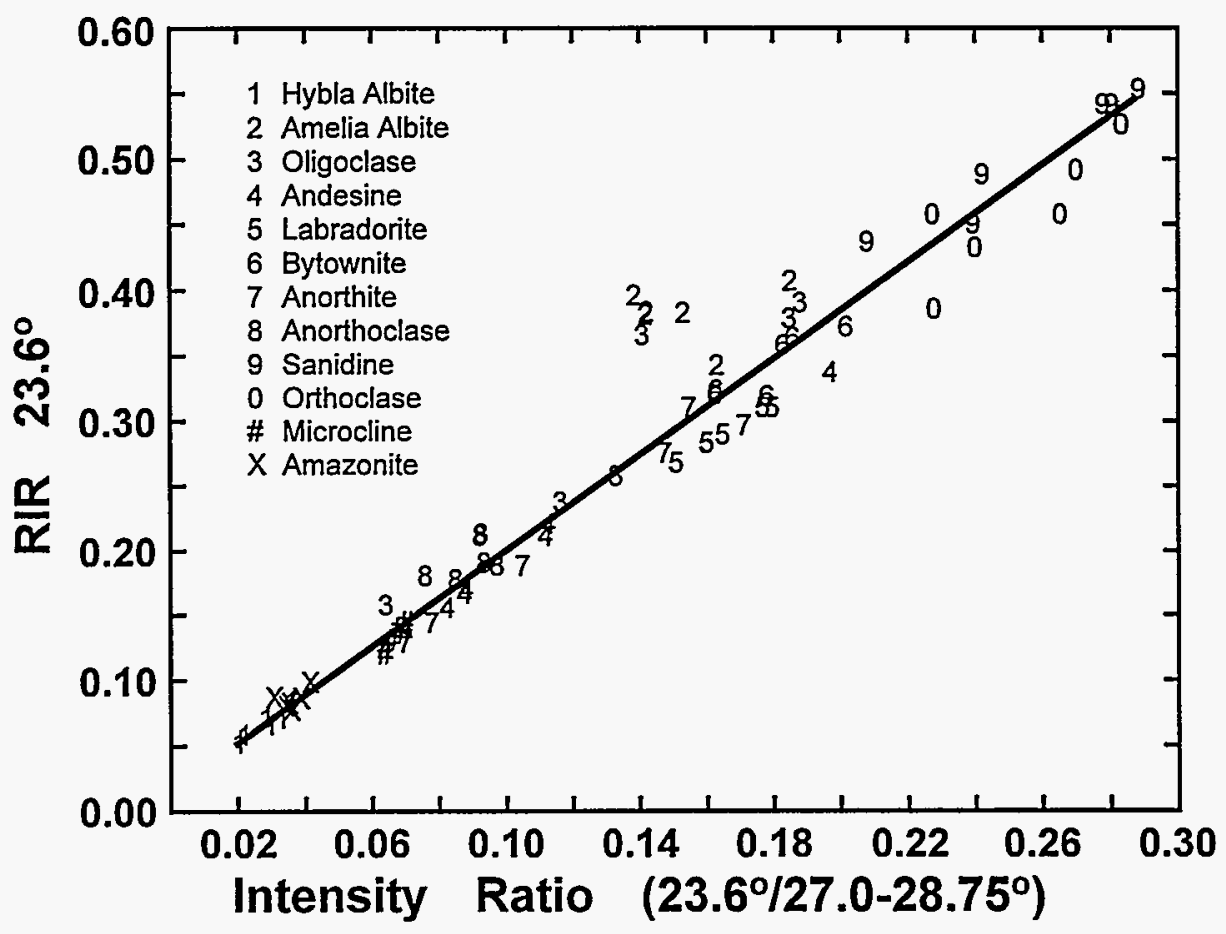

Fig. 3. Relationship between the RIR for the $23.6^{\circ}$ feldspar peak and the intensity ratio of the $23.6^{\circ}$ peak to the sum of the reflections in the $27.0-28.75^{\circ}$ region (CuK $\alpha$ ). 
Table 2. Quantitative X-Ray Diffraction Results for Samples from Drill Hole UE-25 UZ\#16

\begin{tabular}{|c|c|c|c|c|c|c|c|c|c|c|c|c|c|c|c|c|}
\hline SAMPLE & $\begin{array}{c}\text { Depth } \\
(\mathrm{m})\end{array}$ & $\begin{array}{c}\text { Smec- } \\
\text { tite } \\
\end{array}$ & $\begin{array}{c}\text { Steller- } \\
\text { ite }\end{array}$ & $\begin{array}{c}\text { Clino- } \\
\text { ptilolite }^{1}\end{array}$ & $\begin{array}{l}\text { Mor- } \\
\text { denite }\end{array}$ & $\begin{array}{l}\text { Tridy- } \\
\text { mite }\end{array}$ & $\begin{array}{c}\text { Cristo- } \\
\text { balite }\end{array}$ & $\begin{array}{l}\text { Opal- } \\
\text { CT }\end{array}$ & Quartz & $\begin{array}{l}\text { Feld- } \\
\text { spar }\end{array}$ & Glass & $\begin{array}{c}\text { Hema- } \\
\text { tite }\end{array}$ & Mica & $\begin{array}{c}\text { Kaolin- } \\
\text { ite }\end{array}$ & Calcite & Total \\
\hline \multicolumn{3}{|c|}{ Alluvial Fill } & \multicolumn{3}{|c|}{$0.0-12.1$ meters } & & & & & & & & & & & \\
\hline $39.2 / 39.3$ & 12.0 & Trace & --- & -- & -- & $20 \pm 2$ & $15 \pm 2$ & $\cdots$ & $1 \pm 1$ & $59 \pm 8$ & --- & Trace & Trace & --- &.- & $95 \pm 9$ \\
\hline \multicolumn{3}{|c|}{ Tiva Canyon Member } & \multicolumn{3}{|c|}{12.1 - 49.0 meters } & & & & & & & & & & & \\
\hline $54.8 / 55.0$ & 16.8 & $2 \pm 1$ & --- & --- & --- & $4 \pm 1$ & $28 \pm 2$ & -- & $2 \pm 1$ & $61 \pm 9$ & -- & $1 \pm 1$ & -- & --- & $m$ & $98 \pm 9$ \\
\hline $64.7 / 64.9$ & 19.8 & $3 \pm 1$ & --- & $\cdots$ & $-\cdots$ & $7 \pm 1$ & $25 \pm 2$ & $\ldots$ & $4 \pm 1$ & $62 \pm 9$ &.-- & Trace & -- & --- & --- & $101 \pm 99$ \\
\hline $78.6 / 78.9$ & 24.1 & $3 \pm 1$ & $-\cdots$ &.-- & -- & $3 \pm 1$ & $30 \pm 2$ & --- & $1 \pm 1$ & $59 \pm 8$ & --- & Trace & --- & -- & $1 \pm 1$ & $97 \pm 8$ \\
\hline $88.7 / 88.8$ & 27.1 & $2 \pm 1$ & $-\cdots$ & -- & $m$ & $3 \pm 1$ & $31 \pm 2$ & -- & $1 \pm 1$ & $59 \pm 8$ & -- & $3 \pm 1$ &.- & -- & --- & $99 \pm 8$ \\
\hline $102.1 / 102.2$ & 31.2 & $3 \pm 1$ & --- & -- & -- & $3 \pm 1$ & $32 \pm 2$ & -- & Trace & $57 \pm 8$ & $\cdots$ & $1 \pm 1$ & --- & --- & $m$ & $96 \pm 8$ \\
\hline $112.5 / 112.7$ & 34.4 & $3 \pm 1$ & $\cdots$ & -- & -- & $2 \pm 1$ & $32 \pm 2$ & --- & Trace & $58 \pm 8$ & -- & $1 \pm 1$ & -- & -- & $\ldots$ & $96 \pm 8$ \\
\hline $126.0 / 126.2$ & 38.5 & $1 \pm 1$ & -- & $1 \pm 1$ & -- & Trace & $33 \pm 2$ & -- & Trace & $59 \pm 8$ & --- & $1 \pm 1$ & $-\ldots$ & --- & -.- & $95 \pm 8$ \\
\hline $140.2 / 140.3$ & 42.8 & $1 \pm 1$ & --- & -..- & $\ldots$ & Trace & $32 \pm 2$ &.-- & $2 \pm 1$ & $58 \pm 8$ & --- & Trace & -- & --- & --- & $93 \pm 8$ \\
\hline $154.2 / 154.5$ & 47.1 & $8 \pm 2$ & -- & $\ldots$ & - &.-- & $12 \pm 1$ & -- & --- & $17 \pm 2$ & $63 \pm 3$ & Trace & -.. & --- & --- & $37 \pm 3$ \\
\hline $156.1 / 156.2$ & 47.6 & $14 \pm 4$ & --- & --- & --- & --- & Trace & --- & Trace & $4 \pm 1$ & $82 \pm 4$ & -- & -- & $\ldots$ & -- & $100 \pm 4$ \\
\hline \multicolumn{3}{|c|}{ Paintbrush Bedded Tuffs } & \multicolumn{3}{|c|}{49.0 - 63.9 meters } & & & & & & & & & & & \\
\hline $165.1 / 165.3$ & 50.4 & $6 \pm 2$ & --- & --- & -.- & --- & $2 \pm 1$ & $-\cdots$ & $5 \pm 1$ & $16 \pm 2$ & $71 \pm 3$ & $-\cdots$ & Trace & --- & -- & $100 \pm 3$ \\
\hline $167.4 / 167.5$ & 51.1 & $12 \pm 4$ & --- & $\ldots$ & $-\cdots$ &.- & --- & $-\ldots$ & --- & $3 \pm 1$ & $85 \pm 4$ & --- & -- & --- & -- & $100 \pm 4$ \\
\hline $171.3 / 171.5$ & 52.3 & $9 \pm 3$ & $-\cdots$ & --- & --- & --- & $-\cdots$ & $\cdots$ & --- & --- & $91 \pm 3$ & --- & -.. & -- & --- & $100 \pm 3$ \\
\hline $174.9 / 175.1$ & 53.4 & $11 \pm 3$ & --- & -- & -- & -- & $2 \pm 1$ & --- & $1 \pm 1$ & $8 \pm 1$ & $78 \pm 3$ & Trace & --- & --- & $\cdots$ & $100 \pm 3$ \\
\hline $179.3 / 179.4$ & 54.7 & $6 \pm 2$ & --- & --- & --- & -- & $2 \pm 1$ & --- & $7 \pm 1$ & $20 \pm 3$ & $65 \pm 3$ & -- & Trace & --- & --- & $100 \pm 4$ \\
\hline $183.9 / 184.1$ & 56.1 & $18 \pm 5$ & $-\infty$ & -- & --- & --- & $1 \pm 1$ & -- & $2 \pm 1$ & $19 \pm 3$ & $59 \pm 6$ & --- & $1 \pm 1$ & -- & -- & $100 \pm 6$ \\
\hline $188.5 / 188.7$ & 57.5 & $7 \pm 2$ & -- &.-- & -- & $\ldots$ & $1 \pm 1$ & $\cdots$ & Trace & $20 \pm 3$ & $70 \pm 4$ & --- & $1 \pm 1$ & --- & $1 \pm 1$ & $100 \pm 4$ \\
\hline $190.4 / 190.5$ & 58.1 & $16 \pm 5$ & --- & --- & --- & -- & $1 \pm 1$ & -- & $2 \pm 1$ & $19 \pm 3$ & $58 \pm 6$ & --- & $4 \pm 1$ & --- & Trace & $100 \pm 6$ \\
\hline $201.8 / 202.2$ & 61.6 & $9 \pm 3$ & -- & --- & -.- & --- & Trace & --- & $3 \pm 1$ & $19 \pm 3$ & $64 \pm 4$ & Trace & $5 \pm 2$ & -- & Trace & $100 \pm 5$ \\
\hline \multicolumn{6}{|c|}{ Topopah Spring Member $\quad 63.9$ - 366.2 meters } & & & & & & & & & & & \\
\hline $211.4 / 211.6$ & 64.5 & $8 \pm 2$ & $-\cdots$ &.-- & --- & $\ldots$ & Trace & -- & Trace & $19 \pm 3$ & $71 \pm 3$ & $-\cdots$ & $2 \pm 1$ & --- & Trace & $100 \pm 4$ \\
\hline $217.1 / 218.0$ & 66.5 & $18 \pm 5$ & --- & --- & --- & --- & Trace & -- & $1 \pm 1$ & $20 \pm 3$ & $57 \pm 6$ & Trace & $4 \pm 1$ & -- & Trace & $100 \pm 6$ \\
\hline $223.7 / 224.2$ & 68.3 & $9 \pm 3$ & --- & $18 \pm 1$ & -- & --- & $15 \pm 1$ & $-\cdots$ & --- & $55 \pm 9$ & --- & $2 \pm 1$ & $3 \pm 1$ & --- & $2 \pm 1$ & $104 \pm 10$ \\
\hline
\end{tabular}

1 Includes heulandite as clinoptilolite. 
Table 2. Quantitative X-Ray Diffraction Results for Samples from Drill Hole UE-25 UZ\#16 (Continued)

\begin{tabular}{|c|c|c|c|c|c|c|c|c|c|c|c|c|c|c|c|c|}
\hline SAMPLE & $\begin{array}{c}\text { Depth } \\
\text { (m) }\end{array}$ & $\begin{array}{c}\text { Smec- } \\
\text { tite } \\
\end{array}$ & $\begin{array}{c}\text { Steller- } \\
\text { ite }\end{array}$ & $\begin{array}{c}\text { Clino- } \\
\text { ptilolite }\end{array}$ & $\begin{array}{c}\text { Mor- } \\
\text { denite }\end{array}$ & $\begin{array}{l}\text { Tridy- } \\
\text { mite }\end{array}$ & $\begin{array}{c}\text { Cristo- } \\
\text { balite }\end{array}$ & $\begin{array}{l}\text { Opal- } \\
\text { CT }\end{array}$ & Quartz & $\begin{array}{l}\text { Feld- } \\
\text { spar }\end{array}$ & Glass & $\begin{array}{l}\text { Hema- } \\
\text { tite }\end{array}$ & Mica & $\begin{array}{l}\text { Kaolin- } \\
\text { ite }\end{array}$ & Calcite & Total \\
\hline $224.1 / 224.7$ & 68.5 & $6 \pm 2$ & --- & $18 \pm 1$ & --- & --. & $24 \pm 2$ & --- & $2 \pm 1$ & $48 \pm 8$ & -.. & $1 \pm 1$ & $l \pm 1$ & -.. & Trace & $100 \pm 9$ \\
\hline $227.0 / 227.2$ & 69.3 & $5 \pm 2$ & --- & $17 \pm 1$ & --- & $\cdots$ & $34 \pm 2$ & --- & $1 \pm 1$ & $41 \pm 6$ & $-\cdots$ & $1 \pm 1$ & $\cdots$ & -- & -- & $99 \pm 7$ \\
\hline $230.1 / 230.3$ & 70.2 & $1 \pm 1$ & --- & --- & --- & --. & $1 \pm 1$ & --- & Trace & $21 \pm 3$ & $73 \pm 3$ & $2 \pm 1$ & $2 \pm 1$ & --- & ... & $100 \pm 4$ \\
\hline $234.1 / 234.2$ & 71.4 & Trace & $\cdots$ & --- & $\cdots$ & --- & $2 \pm 1$ & --- & Trace & $38 \pm 5$ & $58 \pm 5$ & $-\cdots$ & $2 \pm 1$ & --- & -- & $100 \pm 5$ \\
\hline $249.8 / 250.0$ & 76.2 & $1 \pm 1$ & $-\cdots$ & --. & $-\cdots$ & $3 \pm 1$ & $13 \pm 1$ & --- & -... & $78 \pm 11$ & --- & $1 \pm 1$ & $1 \pm 1$ & -- & -.- & $97 \pm 11$ \\
\hline $269.2 / 269.8$ & 82.2 & $1 \pm 1$ & $\cdots$ & --- & $\cdots$ & $7 \pm 1$ & $11 \pm 1$ & --- & $-\cdots$ & $81 \pm 11$ & --- & $1 \pm 1$ & $1 \pm 1$ & --- & -- & $102 \pm 11$ \\
\hline $290.8 / 291.2$ & 88.8 & $1 \pm 1$ &.-- & --- &.-- & $12 \pm 1$ & $8 \pm 4$ & --- & --- & $77 \pm 11$ & $-\cdots$ & $1 \pm 1$ & $1 \pm 1$ & --- & --- & $100 \pm 12$ \\
\hline $309.1 / 309.5$ & 94.3 & Trace & --- & --- & --- & $13 \pm 1$ & $8 \pm 4$ & -- & --- & $77 \pm 11$ & -.- & $1 \pm 1$ & $1 \pm 1$ & $\cdots$ & $3 \pm 1$ & $103 \pm 12$ \\
\hline $331.7 / 332.2$ & 100.3 & $1 \pm 1$ & --- & --- & -- & $22 \pm 2$ & $5 \pm 3$ & $\ldots$ & --- & $76 \pm 11$ & -.- & $1 \pm 1$ & Trace & --- & $\cdots$ & $105 \pm 12$ \\
\hline $348.7 / 349.7$ & 106.6 & $5 \pm 2$ & $\cdots$ & $\ldots$ & $\cdots$ & $22 \pm 2$ & $8 \pm 4$ & -- & $\cdots$ & $64 \pm 9$ & --- & $1 \pm 1$ & Trace & -- & -- & $100 \pm 10$ \\
\hline $370.2 / 370.7$ & 113.0 & $7 \pm 2$ & --- & --- & --- & $22 \pm 2$ & $8 \pm 4$ & --. & $3 \pm 1$ & $61 \pm 9$ & -- & $1 \pm 1$ & Trace & $\ldots$ &.-- & $102 \pm 10$ \\
\hline $391.6 / 392.0$ & 119.5 & $6 \pm 2$ & --- & $\ldots$ & --- & $11 \pm 1$ & $21 \pm 2$ & $\ldots$ & $4 \pm 1$ & $54 \pm 8$ & --- & $1 \pm 1$ & Trace & --- & -.. & $97 \pm 9$ \\
\hline $412.7 / 413.0$ & 125.9 & $4 \pm 1$ & --. & $\ldots$ & $-\cdots$ & $16 \pm 2$ & $19 \pm 2$ & --- & $2 \pm 1$ & $56 \pm 8$ & --- & $1 \pm 1$ & Trace & --- & --- & $98 \pm 9$ \\
\hline $435.1 / 435.5$ & 132.7 & $4 \pm 1$ & $\cdots$ & --- & $-\cdots$ & $7 \pm 1$ & $28 \pm 2$ & --- & $3 \pm 1$ & $58 \pm 8$ & --- & $1 \pm 1$ & Trace & $\ldots$ & --- & $101 \pm 8$ \\
\hline $458.6 / 458.9$ & 139.9 & $4 \pm 1$ & -- & --- & --- & $10 \pm 1$ & $17 \pm 2$ & --- & $11 \pm 1$ & $56 \pm 8$ & $\ldots$ & $1 \pm 1$ & Trace & --- & $-\cdots$ & $99 \pm 8$ \\
\hline $484.4 / 485.7$ & 148.0 & $4 \pm 1$ & -.- & $\ldots$ & $-\cdots$ & $4 \pm 1$ & $29 \pm 2$ & --- & $5 \pm 1$ & $55 \pm 8$ & --- & $1 \pm 1$ & Trace & -.. & --- & $98 \pm 8$ \\
\hline $509.6 / 510.0$ & 155.5 & $4 \pm 1$ & ... & -.-- & -.. & $3 \pm 1$ & $28 \pm 2$ & --- & $9 \pm 1$ & $54 \pm 8$ & ... & $1 \pm 1$ & Trace & ... & ... & $99 \pm 8$ \\
\hline $533.3 / 533.6$ & 162.6 & $4 \pm 1$ & $\ldots$ & --- & --- & $1 \pm 1$ & $25 \pm 2$ & --- & $11 \pm 1$ & $55 \pm 8$ & $\ldots$ & $1 \pm 1$ & Trace & --. & $\ldots$ & $97 \pm 8$ \\
\hline $552.9 / 553.3$ & 168.7 & $3 \pm 1$ & $\ldots$ & --- & --- & Trace & $16 \pm 1$ & --- & $21 \pm 2$ & $57 \pm 8$ & -... & .... & Trace & --- & -.. & $97 \pm 8$ \\
\hline $577.3 / 577.6$ & 176.1 & $3 \pm 1$ & -- & --- & -- & $1 \pm 1$ & $13 \pm 1$ & --- & $23 \pm 2$ & $57 \pm 8$ & $\ldots$ & Trace & Trace & --- & $\ldots$ & $97 \pm 8$ \\
\hline $602.8 / 603.4$ & 183.9 & $3 \pm 1$ & ... & --- & --- & $3 \pm 1$ & $27 \pm 2$ & --- & $12 \pm 1$ & $57 \pm 8$ & $-\cdots$ & $\cdots$ & Trace & --- & -.. & $102 \pm 8$ \\
\hline $627.9 / 628.2$ & 191.5 & $3 \pm 1$ & Trace & -.. & -.. & $1 \pm 1$ & $26 \pm 2$ & --- & $10 \pm 1$ & $56 \pm 8$ & --- & --- & Trace & -.. & $\ldots$ & $96 \pm 8$ \\
\hline $648.8 / 649.3$ & 197.9 & $4 \pm 1$ & $1 \pm 1$ & $-\cdots$ & $\ldots$ & $4 \pm 1$ & $27 \pm 2$ & $\ldots$ & $6 \pm 1$ & $54 \pm 8$ & --- & $1 \pm 1$ & Trace & $\ldots$ & --- & $97 \pm 9$ \\
\hline $674.2 / 674.7$ & 205.7 & $5 \pm 2$ & -.- & --- & --. & $1 \pm 1$ & $13 \pm 1$ & -. & $27 \pm 2$ & $58 \pm 8$ & --- & --- & Trace & --- & --- & $104 \pm 9$ \\
\hline $701.5 / 701.9$ & 213.9 & $3 \pm 1$ & -.- & ... & $-\cdots$ & $1 \pm 1$ & $5 \pm 2$ & --- & $36 \pm 2$ & $58 \pm 8$ & $\cdots$ & $\ldots$ & Trace & --- & -- & $103 \pm 5$ \\
\hline
\end{tabular}

${ }^{1}$ Includes heulandite as clinoptilolite. 
Table 2. Quantitative X-Ray Diffraction Results for Samples from Drill Hole UE-25 UZ\#16 (Continued)

\begin{tabular}{|c|c|c|c|c|c|c|c|c|c|c|c|c|c|c|c|c|}
\hline SAMPLE & $\begin{array}{c}\text { Depth } \\
(\mathrm{m})\end{array}$ & $\begin{array}{c}\text { Smec- } \\
\text { tite }\end{array}$ & $\begin{array}{c}\text { Steller- } \\
\text { ite }\end{array}$ & $\begin{array}{c}\text { Clino- } \\
\text { ptilolite }\end{array}$ & $\begin{array}{c}\text { Mor- } \\
\text { denite }\end{array}$ & $\begin{array}{l}\text { Tridy- } \\
\text { mite }\end{array}$ & $\begin{array}{c}\text { Cristo- } \\
\text { balite } \\
\end{array}$ & $\begin{array}{c}\text { Opal- } \\
\text { CT }\end{array}$ & Quartz & $\begin{array}{l}\text { Feld- } \\
\text { spar }\end{array}$ & Glass & $\begin{array}{c}\text { Hema- } \\
\text { tite }\end{array}$ & Mica & $\begin{array}{c}\text { Kaolin- } \\
\text { ite }\end{array}$ & Calcite & Total \\
\hline $726.1 / 726.4$ & 221.4 & $1 \pm 1$ & $2 \pm 1$ & --- & --. & $7 \pm 1$ & $10 \pm 1$ & --- & $23 \pm 2$ & $57 \pm 8$ & $\cdots$ & $1 \pm 1$ & Trace & -- & -.- & $101 \pm 9$ \\
\hline $751.4 / 751.7$ & 229.1 & $3 \pm 1$ & -- & --- & $\ldots$ & $1 \pm 1$ & $10 \pm 1$ &.-- & $29 \pm 2$ & $56 \pm 8$ & --. & --- & Trace & -- &.- & $99 \pm 8$ \\
\hline $773.0 / 773.4$ & 235.7 & $1 \pm 1$ & $3 \pm 1$ &.-- &.-- & $3 \pm 1$ & $19 \pm 1$ & -- & $17 \pm 1$ & $56 \pm 8$ & -.. & $1 \pm 1$ & Trace & --- & --- & $100 \pm 8$ \\
\hline $797.0 / 797.2$ & 243.0 & $1 \pm 1$ & $1 \pm 1$ & -- & -.- & $1 \pm 1$ & $23 \pm 2$ & -.- & $14 \pm 1$ & $57 \pm 8$ & $\cdots$ & $1 \pm 1$ & Trace & -.- & -.- & $98 \pm 9$ \\
\hline $820.6 / 820.9$ & 250.2 & $3 \pm 1$ & $1 \pm 1$ & -- & -- & $1 \pm 1$ & $11 \pm 1$ & -- & $26 \pm 2$ & $56 \pm 8$ & $\cdots$ & $1 \pm 1$ & -- & -- & -- & $99 \pm 9$ \\
\hline $843.0 / 843.5$ & 257.1 & $3 \pm 1$ & Trace & $\cdots$ & $\cdots$ & $6 \pm 1$ & $16 \pm 1$ & $\cdots$ & $18 \pm 1$ & $55 \pm 8$ & -- & $1 \pm 1$ & Trace & --- & -- & $99 \pm 8$ \\
\hline $862.4 / 862.7$ & 263.0 & $2 \pm 1$ & $5 \pm 1$ & --. & --- & $5 \pm 1$ & $22 \pm 2$ & --- & $9 \pm 1$ & $55 \pm 8$ & --- & $1 \pm 1$ & $\ldots$ & -..- & --. & $99 \pm 9$ \\
\hline $876.8 / 877.1$ & 267.3 & $3 \pm 1$ & $11 \pm 2$ & -.- & -.. & $4 \pm 1$ & $21 \pm 2$ & -.. & $10 \pm 1$ & $53 \pm 7$ & -.. & $1 \pm 1$ & Trace & --- & -.- & $103 \pm 8$ \\
\hline $896.2 / 896.6$ & 273.3 & $4 \pm 1$ & --- & -- & --- & $6 \pm 1$ & $24 \pm 2$ & --- & $9 \pm 1$ & $54 \pm 8$ & --- & Trace & Trace & $\cdots$ & -.. & $97 \pm 8$ \\
\hline $910.2 / 910.4$ & 277.5 & $6 \pm 2$ & $2 \pm 1$ & -- & -- & $3 \pm 1$ & $18 \pm 1$ & -- & $18 \pm 1$ & $56 \pm 8$ & -- & Trace & Trace & -- & -- & $103 \pm 8$ \\
\hline $925.4 / 925.7$ & 282.2 & $4 \pm 1$ & $5 \pm 1$ & $\cdots$ & -- & $4 \pm 1$ & $26 \pm 2$ & -- & $12 \pm 1$ & $50 \pm 7$ & $\cdots$ & $1 \pm 1$ & --- & -- & $\cdots$ & $102 \pm 8$ \\
\hline $940.0 / 940.4$ & 286.6 & $4 \pm 1$ & $1 \pm 1$ & --- & -- & Trace & $13 \pm 1$ & -- & $27 \pm 2$ & $57 \pm 8$ & $\cdots$ & Trace & Trace & --- & -- & $102 \pm 8$ \\
\hline $954.3 / 954.7$ & 291.0 & $3 \pm 1$ & $2 \pm 1$ & --- &.-- & $3 \pm 1$ & $20 \pm 2$ & -- & $18 \pm 1$ & $52 \pm 7$ & --- & $1 \pm 1$ & Trace & --- & --- & $99 \pm 8$ \\
\hline $970.9 / 971.4$ & 296.1 & $5 \pm 2$ & $2 \pm 1$ & -- & -- & $2 \pm 1$ & $21 \pm 2$ & --- & $17 \pm 1$ & $51 \pm 7$ & -.. & Trace & Trace & -- & $\cdots$ & $98 \pm 8$ \\
\hline $984.2 / 984.8$ & 300.2 & $4 \pm 1$ & $4 \pm 1$ & $-\cdots$ & -- & $2 \pm 1$ & $21 \pm 2$ & $-\cdots$ & $18 \pm 1$ & $52 \pm 7$ & $-\cdots$ & -.. & Trace & --- &.-- & $101 \pm 8$ \\
\hline $1001.0 / 1001.6$ & 305.3 & $5 \pm 2$ & $4 \pm 1$ & --. & --. & $1 \pm 1$ & $16 \pm 1$ & -.- & $22 \pm 2$ & $50 \pm 7$ & --- & -- & Trace & -- & --- & $98 \pm 8$ \\
\hline $1014.9 / 1015.0$ & 309.4 & $6 \pm 2$ & -.- & --- & --- & $5 \pm 1$ & $21 \pm 2$ & $-\cdots$ & $14 \pm 1$ & $53 \pm 7$ & -- & Trace & $1 \pm 1$ & --- & --- & $100 \pm 8$ \\
\hline $1029.2 / 1029.5$ & 313.8 & $5 \pm 2$ & $14 \pm 3$ & $\cdots$ & --- & $3 \pm 1$ & $21 \pm 2$ & --- & $15 \pm 1$ & $45 \pm 6$ & $\cdots$ & $1 \pm 1$ & Trace & $\cdots$ & --- & $104 \pm 7$ \\
\hline $1030.4 / 1030.6$ & 314.1 & $4 \pm 1$ & $8 \pm 2$ & --- & -- & $2 \pm 1$ & $18 \pm 1$ & --- & $20 \pm 2$ & $50 \pm 7$ & -- & Trace & Trace & -- & -.- & $102 \pm 8$ \\
\hline $1047.0 / 1047.3$ & 319.2 & $4 \pm 1$ & $2 \pm 1$ & -- & -- & $2 \pm 1$ & $13 \pm 1$ &.- & $24 \pm 2$ & $55 \pm 8$ & -- & Trace & Trace & $\cdots$ & $\cdots$ & $100 \pm 8$ \\
\hline $1059.6 / 1059.8$ & 323.0 & $5 \pm 2$ & --- & --- & -- & $3 \pm 1$ & $23 \pm 2$ & -- & $15 \pm 1$ & $54 \pm 8$ & -- & Trace & Trace & -- & $\cdots$ & $100 \pm 9$ \\
\hline $1074.8 / 1075.1$ & 327.7 & $4 \pm 1$ & $1 \pm 1$ & --- & --- & $2 \pm 1$ & $17 \pm 1$ & -.. & $21 \pm 2$ & $54 \pm 8$ & -.. & Trace & Trace & --- & -.- & $99 \pm 8$ \\
\hline $1090.4 / 1090.6$ & 332.4 & $4 \pm 1$ & $2 \pm 1$ & --- & $-\cdots$ & $1 \pm 1$ & $15 \pm 1$ & $\ldots$ & $24 \pm 2$ & $53 \pm 7$ & - & Trace & $1 \pm 1$ & --- & $\cdots$ & $100 \pm 8$ \\
\hline $1104.1 / 1104.4$ & 336.6 & $2 \pm 1$ & --- & --- & -- & --- & $8 \pm 3$ & --- & $31 \pm 2$ & $57 \pm 8$ & -- & Trace & Trace & -.. & --- & $98 \pm 9$ \\
\hline
\end{tabular}

${ }^{1}$ Includes heulandite as clinoptilolite. 
Table 2. Quantitative X-Ray Diffraction Results for Samples from Drill Hole UE-25 UZ\#16 (Continued)

\begin{tabular}{|c|c|c|c|c|c|c|c|c|c|c|c|c|c|c|c|c|}
\hline SAMPLE & $\begin{array}{c}\text { Depth } \\
\text { (m) }\end{array}$ & $\begin{array}{c}\text { Smec- } \\
\text { tite }\end{array}$ & $\begin{array}{c}\text { Steller- } \\
\text { ite }\end{array}$ & $\begin{array}{c}\text { Clino- } \\
\text { ptilolite }^{1}\end{array}$ & $\begin{array}{l}\text { Mor- } \\
\text { denite }\end{array}$ & $\begin{array}{c}\text { Tridy- } \\
\text { mite }\end{array}$ & $\begin{array}{c}\text { Cristo- } \\
\text { balite }\end{array}$ & $\begin{array}{c}\text { Opal- } \\
\text { CT }\end{array}$ & Quartz & $\begin{array}{l}\text { Feld- } \\
\text { spar }\end{array}$ & Glass & $\begin{array}{c}\text { Hema- } \\
\text { tite }\end{array}$ & Mica & $\begin{array}{c}\text { Kaolin } \\
\text { ite }\end{array}$ & Calcite & Total \\
\hline $1113.1 / 1113.2 \mathrm{a}$ & 339.3 & $73 \pm 22$ & --- & $4 \pm 1$ & --- & -- & $10 \pm 1$ & --- & Trace & $10 \pm 1$ & $-\cdots$ & -- & Trace & --- & --. & $97 \pm 22$ \\
\hline $1113.0 / 1113.2 b$ & 339.3 & $5 \pm 2$ & --- & --- & --- & $-\cdots$ & $7 \pm 1$ & --- & $1 \pm 1$ & $14 \pm 2$ & $73 \pm 3$ & -- & Trace & --- & --- & $100 \pm 3$ \\
\hline $1135.3 / 1135.6$ & 346.1 & $3 \pm 1$ & -- & -- & -- & --- & $-\cdots$ & $9 \pm 2$ & $1 \pm 1$ & $7 \pm 1$ & $80 \pm 2$ & -- & Trace & -- & -- & $100 \pm 3$ \\
\hline $1149.2 / 1149.5$ & 350.4 & --- & --- & --- & -- & --- & --- & $10 \pm 2$ & $1 \pm 1$ & $7 \pm 1$ & $82 \pm 2$ & --- & Trace & $\cdots$ & --- & $100 \pm 2$ \\
\hline $1157.4 / 1157.6$ & 352.8 & $1 \pm 1$ & $-\cdots$ & $-\cdots$ & $\cdots$ & $-\cdots$ & --- & $12 \pm 3$ & $1 \pm 1$ & $10 \pm 1$ & $75 \pm 3$ & $-\cdots$ & Trace & $\cdots$ & $1 \pm 1$ & $100 \pm 4$ \\
\hline $1166.5 / 1167.0$ & 355.7 & $2 \pm 1$ & --- & $10 \pm 1$ & $-\infty$ & --- & -- & $20 \pm 6$ & $2 \pm 1$ & $17 \pm 3$ & $49 \pm 6$ & $\cdots$ & Trace & --- & -- & $100 \pm 7$ \\
\hline $1179.8 / 1180.5$ & 359.8 & $1 \pm 1$ & --- & $80 \pm 6$ & $\cdots$ & --- & $\cdots$ & $13 \pm 3$ & $3 \pm 1$ & $8 \pm 2$ & $\cdots$ & -.. & Trace & -- & -- & $105 \pm 7$ \\
\hline $1190.2 / 1191.2$ & 363.1 & $6 \pm 2$ & --- & $83 \pm 6$ & $2 \pm 1$ & $-\cdots$ & $\cdots$ & $6 \pm 1$ & $1 \pm 1$ & $4 \pm 1$ & --- & --- & Trace & -.- & -.- & $102 \pm 7$ \\
\hline \multicolumn{3}{|c|}{ Calico Hills Formation } & \multicolumn{3}{|c|}{366.2 - 452.6 meters } & & & & & & & & & & & \\
\hline $1202.8 / 1203.1$ & 366.7 & $1 \pm 1$ & $-\cdots$ & $66 \pm 9$ & $12 \pm 4$ & $\cdots$ & --- & $14 \pm 3$ & $3 \pm 1$ & $6 \pm 2$ & --- & --- & --- & $\cdots$ & $-\cdots$ & $102 \pm 11$ \\
\hline $1215.7 / 1216.0$ & 370.6 & $1 \pm 1$ & $-\cdots$ & $72 \pm 9$ & $11 \pm 4$ & $\cdots$ & --- & $12 \pm 3$ & $3 \pm 1$ & $7 \pm 2$ & $\cdots$ & -- & Trace & $-\cdots$ & -- & $106 \pm 11$ \\
\hline $1235.5 / 1235.7$ & 376.6 & $1 \pm 1$ & --- & $69 \pm 8$ & $7 \pm 2$ & --- & --- & $18 \pm 4$ & $3 \pm 1$ & $7 \pm 2$ & $-\cdots$ & -- & Trace & $\cdots$ & $\cdots$ & $105 \pm 9$ \\
\hline $1256.6 / 1256.9$ & 383.1 & $8 \pm 2$ & $-\cdots$ & $72 \pm 5$ & --- & -.. & --- & $10 \pm 3$ & $3 \pm 1$ & $12 \pm 3$ & --- & --- & Trace & $\cdots$ & $\cdots$ & $105 \pm 7$ \\
\hline $1261.0 / 1261.2 \mathrm{a}$ & 384.4 & $1 \pm 1$ & $-\cdots$ & $66 \pm 5$ & -- & $-\cdots$ & --- & $22 \pm 6$ & $3 \pm 1$ & $11 \pm 2$ & --- & --- & Trace & -- & --- & $103 \pm 8$ \\
\hline $1261.0 / 1261.2 b$ & 384.4 & --- & --- & $8 \pm 1$ & $-\cdots$ & $25 \pm 3$ & $10 \pm 2$ & --- & $8 \pm 1$ & $52 \pm 8$ & $\cdots$ & $1 \pm 1$ & Trace & -- & --- & $104 \pm 9$ \\
\hline $1275.7 / 1276.0$ & 388.9 & $3 \pm 1$ & --- & $54 \pm 6$ & $8 \pm 3$ & --- & $-\cdots$ & $18 \pm 5$ & $6 \pm 1$ & $13 \pm 3$ & --- & --- & --- & --- & $\cdots$ & $102 \pm 9$ \\
\hline 1300.3 & 396.3 & $9 \pm 3$ &.-- & $58 \pm 6$ & $5 \pm 2$ & $\cdots$ & -- & $20 \pm 5$ & $5 \pm 1$ & $10 \pm 2$ & --- & --- & --- & $\cdots$ & --- & $107 \pm 9$ \\
\hline $1305.0 / 1305.3$ & 397.9 & $2 \pm 1$ & $-\cdots$ & $60 \pm 7$ & $9 \pm 3$ & $\cdots$ & --- & $19 \pm 5$ & $5 \pm 1$ & $11 \pm 3$ & --- & --- & $\cdots$ & $\cdots$ & -- & $106 \pm 10$ \\
\hline $1312.0 / 1312.4$ & 400.0 & $3 \pm 1$ & $\cdots$ & $54 \pm 7$ & $12 \pm 4$ & $\cdots$ & --- & $18 \pm 5$ & $4 \pm 1$ & $11 \pm 3$ & -- & --- & -- & $\cdots$ & --- & $102 \pm 10$ \\
\hline $1335.4 / 1336.2$ & 407.3 & $1 \pm 1$ & $\cdots$ & $38 \pm 5$ & $11 \pm 3$ & --- & -- & $26 \pm 8$ & $9 \pm 1$ & $19 \pm 4$ & --- & --- & --- & $\cdots$ & --- & $104 \pm 11$ \\
\hline $1342.7 / 1343.0$ & 409.4 & $3 \pm 1$ & --- & $55 \pm 9$ & $16 \pm 5$ & --- & --- & $19 \pm 5$ & $3 \pm 1$ & $10 \pm 3$ & --- & --- & --- & -- & --- & $106 \pm 12$ \\
\hline $3 / 1362.7$ & 415.4 & $3 \pm 1$ & --- & $52 \pm 7$ & $13 \pm 4$ & --- & $\cdots$ & $17 \pm 4$ & $4 \pm 1$ & $11 \pm 3$ & $\cdots$ & $\cdots$ & -- & --- & $\cdots$ & $100 \pm 10$ \\
\hline $1379.3 / 1379.5$ & 420.5 & $3 \pm 1$ & $-\cdots$ & $60 \pm 8$ & $9 \pm 3$ & -- & --- & $21 \pm 5$ & $4 \pm 1$ & $9 \pm 2$ & $-\cdots$ & --- & $-\cdots$ & --- & -.- & $106 \pm 10$ \\
\hline $1417.5 / 1418.0$ & 432.2 & $2 \pm 1$ & --- & $58 \pm 7$ & $8 \pm 3$ & $\cdots$ & --- & $19 \pm 5$ & $4 \pm 1$ & $11 \pm 3$ & --- & --- & --- & $\cdots$ & --- & $102 \pm 10$ \\
\hline 1441.3/1441.8 & 439.5 & $2 \pm 1$ & --- & $65 \pm 9$ & $12 \pm 4$ & --- & $\cdots$ & $17 \pm 4$ & $3 \pm 1$ & $6 \pm 2$ & --- & $\cdots$ & -- & -- & --. & $105 \pm 11$ \\
\hline $1460.2 / 1460.7$ & 445.2 & $5 \pm 2$ & $-\cdots$ & $42 \pm 4$ & $4 \pm 1$ & $-\cdots$ & --- & $27 \pm 8$ & $8 \pm 1$ & $18 \pm 4$ & $\cdots$ & -.- & $\cdots$ & --- & -- & $104 \pm 10$ \\
\hline
\end{tabular}

${ }^{1}$ Includes heulandite as clinoptilolite. 
Table 2. Quantitative X-Ray Diffraction Results for Samples from Drill Hole UE-25 UZ\#16 (Continued)

\begin{tabular}{|c|c|c|c|c|c|c|c|c|c|c|c|c|c|c|c|c|}
\hline SAMPLE & $\begin{array}{c}\text { Depth } \\
\text { (m) }\end{array}$ & $\begin{array}{c}\text { Smec- } \\
\text { tite }\end{array}$ & $\begin{array}{c}\text { Steller- } \\
\text { ite }\end{array}$ & $\begin{array}{c}\text { Clino- } \\
\text { ptilolite }\end{array}$ & $\begin{array}{c}\text { Mor- } \\
\text { denite }\end{array}$ & $\begin{array}{l}\text { Tridy- } \\
\text { mite }\end{array}$ & $\begin{array}{c}\text { Cristo- } \\
\text { balite }\end{array}$ & $\begin{array}{c}\text { Opal- } \\
\text { CT }\end{array}$ & Quartz & $\begin{array}{l}\text { Feld- } \\
\text { spar }\end{array}$ & Glass & $\begin{array}{l}\text { Hema- } \\
\text { tite }\end{array}$ & Mica & $\begin{array}{c}\text { Kaolín- } \\
\text { ite }\end{array}$ & Calcite & Total \\
\hline $1476.7 / 1477.0$ & 450.2 & $5 \pm 2$ & $\ldots$ & $40 \pm 3$ & -..- & -.. & --- & $20 \pm 7$ & $11 \pm 1$ & $27 \pm 5$ & --- & -- & $1 \pm 1$ & --. & -.. & $104 \pm 9$ \\
\hline \multicolumn{3}{|c|}{ Prow Pass Member } & \multicolumn{3}{|c|}{452.6 meters to bottom } & & & & & & & & & & & \\
\hline $1498.4 / 1498.9$ & 456.9 & --.- & --- & ...- & $8 \pm 2$ &.-- & $20 \pm 2$ & --. & $17 \pm 1$ & $48 \pm 9$ &.-- & $1 \pm 1$ & --- & $4 \pm 1$ & $\ldots$ & $98 \pm 10$ \\
\hline $1521.7 / 1522.2$ & 464.0 & $3 \pm 1$ & -.- & -..- & $\ldots$ & -.. & --. & --- & $41 \pm 2$ & $54 \pm 8$ & -.- & $1 \pm 1$ & Trace & $2 \pm 1$ & ... & $101 \pm 8$ \\
\hline $1539.3 / 1539.7$ & 469.3 & $4 \pm 1$ & --. & --. & --- & --- & $-\cdots$ & $-\ldots$ & $41 \pm 2$ & $56 \pm 8$ & $\cdots$ & $1 \pm 1$ & Trace & --- & -.. & $102 \pm 8$ \\
\hline $1561.2 / 1561.7$ & 476.0 & $4 \pm 1$ & --- & --. & --- & -- & $\cdots$ & --- & $39 \pm 2$ & $58 \pm 8$ & --- & $\cdots$ & $1 \pm 1$ & --- & --- & $102 \pm 8$ \\
\hline $1579.1 / 1579.9$ & 481.6 & $3 \pm 1$ & -.. & --. & -- & $\cdots$ & -- & --- & $38 \pm 2$ & $59 \pm 8$ & -- & Trace & Trace & --- & --- & $100 \pm 8$ \\
\hline $1600.2 / 1600.4$ & 487.8 & $4 \pm 1$ & --- & --- & -- & --- & $\cdots$ & -- & $39 \pm 2$ & $58 \pm 8$ & --- & Trace & Trace & --- & -- & $101 \pm 4$ \\
\hline \multicolumn{3}{|c|}{ Static Water Level } & \multicolumn{2}{|c|}{489.2 meters } & & & & & & & & & & & & \\
\hline $1618.7 / 1619.2$ & 493.5 & $3 \pm 1$ & -.- & -.- & --. & --- & $14 \pm 1$ & -- & $24 \pm 2$ & $62 \pm 9$ & -- & --- & Trace & -- & --- & $103 \pm 9$ \\
\hline $1638.7 / 1639.2$ & 499.6 & $7 \pm 2$ & --- & -.. & --- & --- & $15 \pm 1$ & --- & $20 \pm 2$ & $55 \pm 8$ & -.- & --- & $1 \pm 1$ & --. & --- & $98 \pm 9$ \\
\hline $1655.5 / 1656.0$ & 504.8 & $6 \pm 2$ & -- & --. & --- & --- & $27 \pm 2$ & --- & $11 \pm 1$ & $56 \pm 8$ & $\cdots$ & --- & -.- & -.. & --- & $100 \pm 9$ \\
\hline $1683.5 / 1684.0$ & 513.3 & $3 \pm 1$ & --- & $42 \pm 4$ & --- & ... & -. & $28 \pm 10$ & $5 \pm 1$ & $28 \pm 5$ & -.- & -.- & -.. & --- & --- & $106 \pm 12$ \\
\hline
\end{tabular}

${ }^{1}$ Includes heulandite as clinoptilolite. 


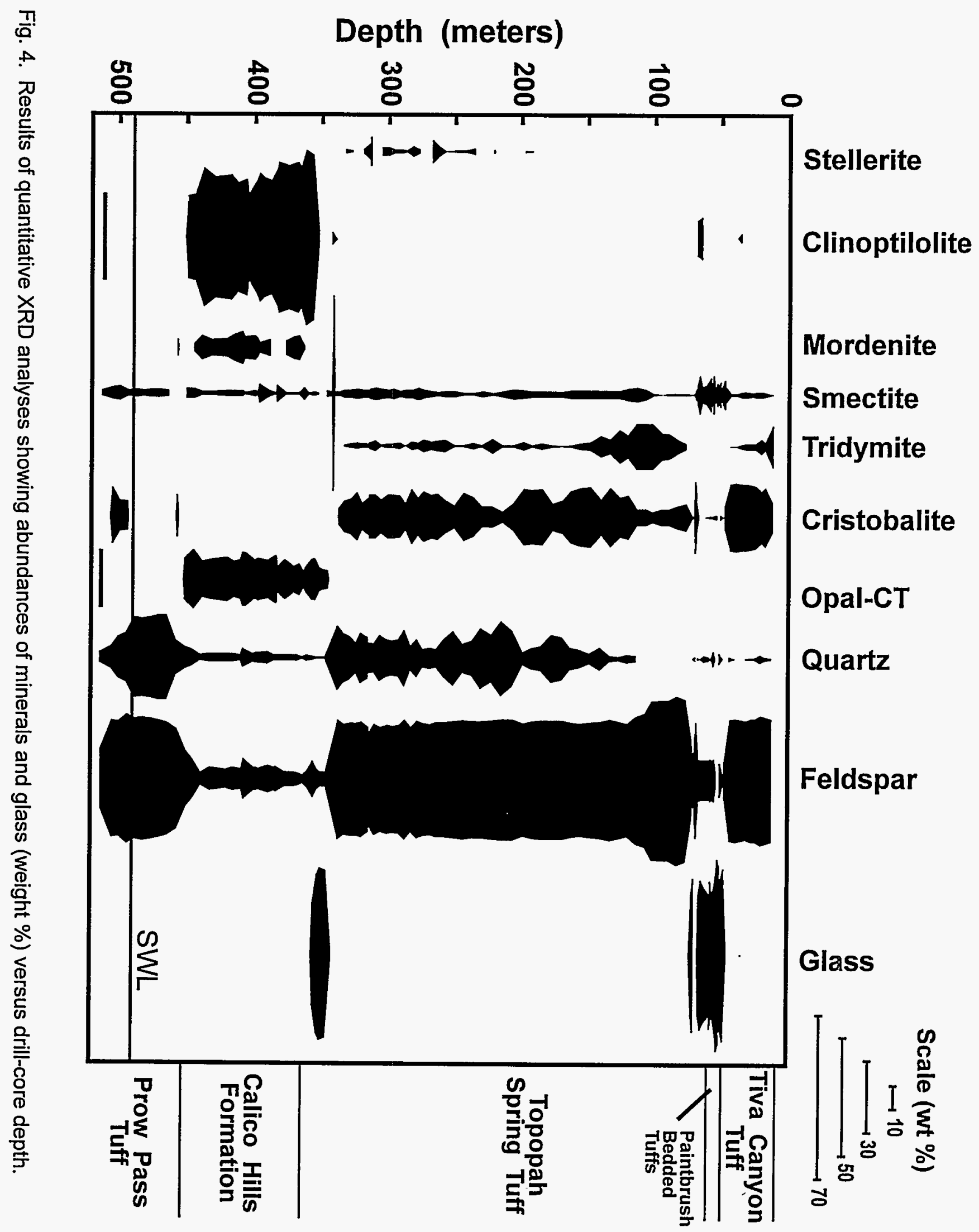


to determine if the structure had collapsed as expected for heulandite (Mumpton 1960; Alietti 1972). Significant collapse occurred upon heating to $400^{\circ} \mathrm{C}$, and a small amount of collapsed heulandite " $\mathrm{B}$ " was observed in the patterns. Heating to $550^{\circ} \mathrm{C}$ produced slightly more collapse and destruction of the heulandite " $\mathrm{B}$ " observed in the $400^{\circ} \mathrm{C}$ samples. Although heating produced significant collapse, the structure was not completely destroyed below $350^{\circ} \mathrm{C}$ as expected for a "type 1" heulandite (Mumpton 1960). Likewise, the zeolite cannot be a pure clinoptilolite which should remain stable to temperatures in excess of $700^{\circ} \mathrm{C}$. The amount of collapse that was observed suggests that the zeolite in these samples is either a mixture of heulandite with minor clinoptilolite, or it is structurally and compositionally an intermediate "type 2" heulandite (Alietti 1972).

Results of quantitative XRD for the middle portion of the drill core show that the main rock types in this interval consist of (1) devitrified Topopah Spring Tuff to $339 \mathrm{~m}$ depth and (2) the lower vitrophyre of the Topopah Spring Tuff to $355 \mathrm{~m}$ depth. Cristobalite, hematite, and smectite were fairly ubiquitous throughout the devitrified Topopah Spring Tuff in UE-25 UZ\#16. Although tridymite occurs throughout this interval, it is most abundant in the upper portion. Quartz is not present in the upper portion of this interval, but occurs in the lower portions in tuffs with reduced tridymite abundance. Mn-oxides were found to coat fractures in the upper portion of the Topopah Spring Tuff, and preliminary analysis of a coating at $162.6 \mathrm{~m}$ revealed the presence of rancieite. Additional analyses of fracture coatings are contained in Appendix I.

Of particular interest in this interval, the zeolite mineral stellerite is common between 191.5 and 332.4 meters in depth, in concentrations of up to $14 w t \%$ (avg. $\sim 3 w t \%$ ). The occurrence of stellerite in the bulk rock is somewhat unusual since it previously has been found only in fractures from drill holes USW G-1, G-2, and UE-25a\#1 (Carlos et al. in press). Stellerite may exist in the bulk rock in other drill holes, but it may not have been previously discovered because of the limited sampling that was conducted in the regions where stellerite may exist (sample spacings in previous studies were typically $\sim 12 \mathrm{~m}$ in this interval). However, this seems unlikely because the extent of the stellerite zone in UE-25 UZ\#16 is so great (141 $\mathrm{m}$ ) that a zone of comparable dimensions would not have been missed in the previously analyzed cores. Another complication in recognizing the presence of stellerite is the significant peak overlaps that occur between stellerite and clinoptilolite, mordenite, cristobalite, and feldspar to the extent that the presence of stellerite can be easily masked by these other phases. Inspection of XRD patterns for selected samples listed in Bish and Chipera (1989) suggests that stellerite may occur in small amounts $(<5 \%)$ in several bulk rock samples in UE$25 a \# 1$ and USW-G1, although the zeolite would have to be separated and concentrated using heavy liquids to confirm its occurrence. Our present assessment of the stellerite data from UE$25 \mathrm{UZ \# 16}$ is that both the thickness of the stellerite zone and the sporadically high abundances 
of stellerite within this zone make the lower Topopah Spring Tuff in this core distinctively different from earlier analyzed cores.

While conducting a separation/purification of stellerite from sample UE-25 UZ\#16-1029 $(313.8 \mathrm{~m})$, a small amount of a magnetic oxide phase was also concentrated from the bulk powder with the use of a hand magnet. This sample was found to be composed of $\sim 85 \%$ magnetite with $\sim 15 \%$ hematite. A Rietveld refinement was conducted to obtain the unit-cell parameters for the magnetite, which when plotted on the chart of Lindsley (1976), showed that the magnetite was $\sim 80 \% \pm 10 \% \mathrm{Fe}_{3} \mathrm{O}_{4}$ end member with only slight oxidation to the $\gamma \mathrm{Fe}_{2} \mathrm{O}_{3}$ (maghemite) composition.

The lower vitrophyre of the Topopah Spring Tuff in UE-25 UZ\#16 is approximately 17 meters thick. Its upper surface has been extensively altered to form a devitrified/vitric transition zone which contains localized clay rich regions ( $75 \%$ smectite) with minor zeolitization. Within the lower vitrophyre, from 352 to $353 \mathrm{~m}$ depth, there is a breccia zone containing calcite. The quantitative XRD data for this breccia zone in Table 2 represent a sample with little calcite; portions of the breccia, however, are filled with abundant coarse-grained calcite. Further studies of the calcite fillings in fractures are being pursued to understand the nature of ongoing fracture transport at Yucca Mountain (Vaniman 1993).

Results of quantitative XRD for the bottom portion of the drill core show that the main rock types in this interval consist of (1) a thin vitric/zeolitic transition zone at $\sim 355 \mathrm{~m}$ depth, (2) the zeolitized bottom of the Topopah Spring Tuff at $366 \mathrm{~m}$, underlain by (3) zeolitized Calico Hills Formation to $\sim 460 \mathrm{~m}$, (4) devitrified Prow Pass Tuff to $\sim 510 \mathrm{~m}$, and (5) at the very bottom of the core is a single sample of zeolitic Prow Pass Tuff (hole T.D. $=514 \mathrm{~m}$ ). Although quartz is ubiquitous in this interval, its abundance is significantly reduced in the zeolitic portions where opal-CT is the authigenic silica polymorph. The zeolitic tuff contains abundant clinoptilolite, often accompanied by mordenite and minor smectite. The devitrified portions of the Prow Pass Tuff are composed of feldspar and quartz, with several sections containing cristobalite. In addition, kaolinite was found at the top the of the devitrified Prow Pass Tuff. One sample of Calico Hills Formation at $384.4 \mathrm{~m}$ was found to contain two significantly contrasting light and dark portions. XRD analyses showed the light portions to be zeolitic tuff whereas the dark portions are devitrified tuff. The dark, devitrified portions are lithic inclusions that were incorporated during deposition of the vitric tuff. Subsequent zeolitic alteration affected the vitric matrix but not the clasts of devitrified tuff. 


\section{DISCUSSION}

\section{Limited Alteration in Vitric Tuffs above the Topopah Spring Tuff}

One of the findings of this study is the relative simplicity of the mineral stratigraphy associated with the complex textural/lithologic variations within the nonwelded tuffs between the Tiva Canyon and Topopah Spring Tuffs. In essence, this nonwelded interval in UE-25 UZ\#16 consists of only two mineralogic units: an upper nonwelded glassy unit of $\sim 21.4 \mathrm{~m}$ (70 ft) thickness and a lower nonwelded devitrified/zeolitic unit of $\sim 3 \mathrm{~m}(10 \mathrm{ft})$ thickness. The simplicity of the mineral stratigraphy is an important observation, indicating the absence of multiple paleoalteration horizons within the nonwelded tuffs.

The restriction of zeolites to the $\sim 3 \mathrm{~m}$ devitrified interval above the upper vitrophyre of the Topopah Spring Tuff, and the identification of a heulandite component in these zeolites associated with abundant cristobalite, may indicate a very early episode of zeolitization during initial heat loss from the underlying vitrophyre. This interpretation is in accord with studies of the lower vitrophyre of the Topopah Spring Tuff, where the formation of heulandite at the margin of the vitrophyre is related to the moderate-temperature events that occurred with latestage cooling of the tuff (Levy and O'Neil 1989). One of the inferences that can be drawn from this interpretation is that the zeolitization observed above the upper vitrophyre of the Topopah Spring Tuff in UE-25 UZ\#16 does not represent any significant accumulation of water over the last 12 million years. This inference should be considered critically in further studies of mineralogy from this interval, for although the occurrences of heulandite-above-glass are similar in both the lower and upper Topopah Spring vitrophyres, the alteration setting is quite different. Heulandite formation in the lower vitrophyre is associated with passage from the overlying devitrification front into the vitrophyre, whereas the heulandite that formed above the upper vitrophyre marks a boundary between two vitric zones (welded and nonwelded) with markedly different porosity and transmissivity. It is also possible that the formation of this zeolitic zone was favored by the presence of minor amounts of perched water captured due to the low permeability of the underlying vitrophyre. Whatever the timing and mode of heulandite formation above the upper vitrophyre, the $3 \mathrm{~m}$ vitrophyre itself is remarkably unaltered, indicating little water/rock interaction in this relatively impermeable interval since tuff emplacement.

Mineralogic evidence of ongoing infiltration may best be found in the potentially ongoing alteration of glass to smectite and in the minor but significant accumulation of calcite in the lower part of the nonwelded interval (57-69 $\mathrm{m}$ depth). It is important to note that the quantities of smectite and calcite that occur in this interval vary markedly across Yucca Mountain, with up to $40 \%$ calcite and $40 \%$ smectite occurring to the north in USW G-2, and 
$40 \%$ smectite but only a trace of calcite occurring in UE-25a\#1. A possible implication of this variability is that those portions of the mountain where recharge has been greatest might be delineated by examining the extent of underlying alteration in the nonwelded tuffs between the Tiva Canyon and Topopah Spring Tuffs. This implication deserves serious consideration.

\section{Distributions of Silica Phases}

The variability of silica polymorph distribution throughout the unsaturated zone reveals a stratigraphy of minerals capable of generating widely varying silica activity in water/rock interactions. Earlier studies (Bish et al. 1984) of core samples have shown a crude stratigraphy of silica polymorphs in the Topopah Spring Tuff, with tridymite concentrated in the upper portions of the devitrified tuff (principally concentrated in the quartz-latite portion that was erupted from a significantly hotter part of the magma chamber than the rhyolite; see Lipman 1971) and with quartz restricted to the lower, rhyolitic portions where tridymite is only a minor phase. Cristobalite is ubiquitous throughout the Topopah Spring Tuff. Examination of the UE25 UZ\#16 data (Table 2, Fig. 4) suggests that although this stratigraphic model continues to be upheld, the lower $86 \%$ of the Topopah Spring devitrified zone contains all three of these crystalline silica polymorphs. Small amounts of tridymite (avg. 2\%) and larger amounts of cristobalite (avg. 18\%) occur throughout the rhyolitic portion of the Topopah Spring Tuff in UE25 UZ\#16. Thus, even at the potential repository horizon, both tridymite and particularly cristobalite may be present to provide relatively soluble silica for dissolution, transport, and deposition under repository thermal conditions.

The distribution of the least-stable silica form in Yucca Mountain cores, opal-CT, is similar to that noted in previous core samples (Bish and Chipera 1989). The occurrence of this phase is strongly correlated with stratified occurrences of zeolites (clinoptilolite/mordenite), where it averages $18 \%$ of zeolitic rocks, and with the lower vitrophyre of the Topopah Spring Tuff, where it averages $10 \%$ of these glassy rocks. The occurrence of opal-CT in the. lower vitrophyre is not universal (e.g., absent in core UE-25a\#1; Bish and Chipera 1989). Moreover, opal-CT has not been found in the upper vitrophyre, although small amounts of cristobalite occur there (1-2\% in UE-25 UZ\#16 and in trace amounts in other cores near the exploration block). Presence or absence of opal in glassy tuffs is important to note, suggesting different environments of alteration. As with the potentially different origins of heulandite in upper and lower vitrophyres, the silicification of the lower vitrophyre and not of the upper vitrophyre point to different alteration regimes. It is possible that alteration was predominantly associated with the advancement of a devitrification front into the lower vitrophyre, whereas alteration may be largely associated with "ponding" above the upper margin of the upper vitrophyre. 
Stellerite in the Lower Half of the Devitrified Topopah Spring Tuff

The occurrence of stellerite provides information on the geochemical conditions that existed during its formation. Chipera et al. (in press) used representative chemical formulas and estimated thermodynamic data to model the conditions under which the various zeolite species at Yucca Mountain formed. Chemical analyses of modern Yucca Mountain waters using the modern silica activities were found to plot within the predicted clinoptilolite stability field (Fig. 5). The stability field of stellerite with respect to clinoptilolite and mordenite grows as the $\mathrm{Ca}$ concentration of the system increases or as the activity of silica decreases. In UE-25 UZ\#16, stellerite is found coexisting with cristobalite whereas clinoptilolite and mordenite coexist with opal-CT. This is consistent with the thermodynamic calculations of Chipera et al. (in press) in which decreasing the silica activity to cristobalite saturation shifts the clinoptilolitemordenite-stellerite stability fields so that present-day water compositions at Yucca Mountain fall within the stability field for stellerite. Localized increase in Ca concentrations would also result in zeolites forming in the stability region for stellerite (Fig. 5).

The wide zone of stellerite distribution and the locally high abundances of stellerite in the devitrified Topopah Spring Tuff contrast with the absence of matrix-associated zeolites in this horizon elsewhere at Yucca Mountain. The occurrence of stellerite cannot be related to a regional paleo-water table since it occurs in or above intervals that contain both glass and tridymite, phases that with rare exceptions do not occur below the water table (Levy 1991). It is possible that the location of UE-25 UZ\#16 within the imbricate fault zone at the eastern margin of the exploration block is a factor in this zeolitization of a devitrified tuff. Zeolite-forming fluids, originating at the surface, may have been introduced into this horizon from nearby faults. Stellerite occurs at Yucca Mountain predominantly in association with faults and fractures (Carlos et al. in press). The holes drilled in this vicinity prior to UZ\#16 provided only cuttings, samples that would "average out" any intermittent spikes of zeolite concentration and therefore provide a poor comparison. Future cored holes will be needed to determine the distribution of stellerite in occurrences such as this. This determination is important, because zeolitization of the potential repository horizon will affect the assessment of waste retardation closest to the repository and the assessment of repository thermal impact on the host rock.

\section{The Zeolitization Front and Possible Paleo-Water Tables}

Changes in past elevations in SWL have implications for possible changes in repository-to-SWL distance under possibly wetter climates in the future. One of the potential mineralogic indicators of past elevations in SWL beneath the exploration block is the distance between the top of the first major zeolitized horizon and the present SWL. Figure 6 summarizes some estimates for this distance from several drill holes, including the current data 


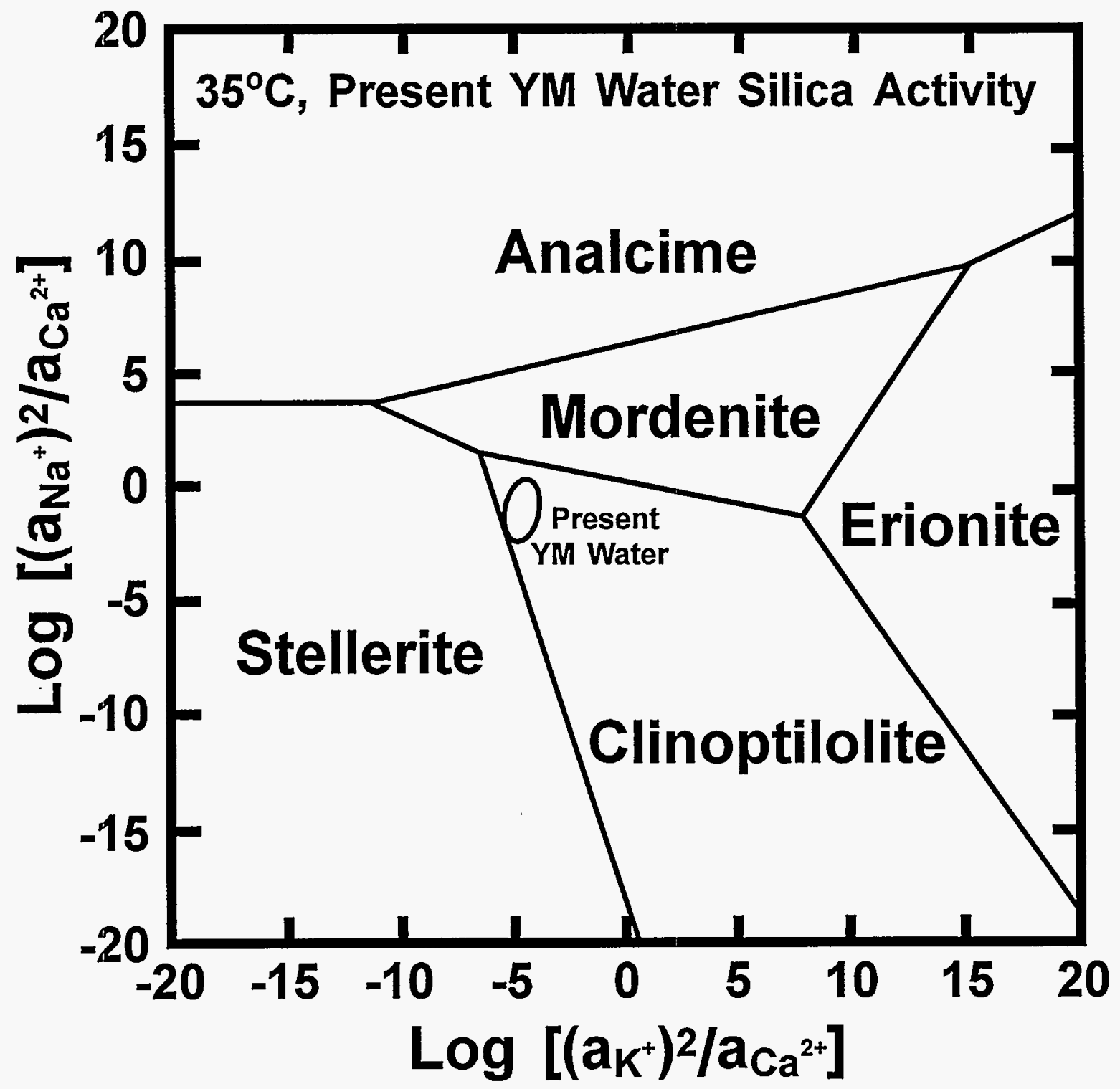

Fig. 5. Log activity diagram for the zeolite phases found at Yucca Mountain calculated using estimated thermodynamic data and representative chemical analyses of the minerals (from Chipera et al. in press). The calculations were constrained using a representative modern Yucca Mountain water value for silica activity and a temperature of $35^{\circ} \mathrm{C}$ (obtained from Kerrisk 1987). Increasing the temperature for the calculation to $125^{\circ} \mathrm{C}$ did not change the diagram significantly. Modern Yucca Mountain water cation ratios for $\mathrm{Ca}-\mathrm{Na}-\mathrm{K}$ show the waters plot within the clinoptilolite stability field although only minor changes to the system would be required to stabilize either mordenite or stellerite. 
from UE-25 UZ\#16. Ideally, a direct correlation between the occurrence of the top of this horizon of abundant zeolitization and an ancient SWL would be seen in all drill holes as a surface occurring at a relatively fixed elevation above the present SWL. However, there are several reasons (Levy 1991) for this not to be the case:

(1) Even if the SWL were to rise, abundant zeolitization would not occur to the height of saturation if appropriate parent material (porous, nonwelded vitric tuff) were not present. This can account for the small apparent elevations in UE-25a\#1, USW H-6, USW WT-1, and UE-25p\#1.

(2) More than one event of rise in the SWL can result in variable elevations of the zeolitization horizon because of possible tectonic offsets in parts of the exploration block between the timing of ancient and more recent rises in the SWL.

(3) The present SWL is relatively flat beneath the exploration block but rises sharply to the north and west (Ervin et al. 1993). Similar variable elevations may occur in an ancient SWL, but not necessarily in the same locations as in the present SWL.

(4) Zeolitization can occur in unsaturated rocks, especially in situations of possible capillary draw, perched water, or lateral transmission from features that may be significant sources of recharge during pluvial episodes.

(5) Widely spaced sample collections, and in particular the need to rely on cuttings or intermittent core/sidewall samples in all $\mathrm{H}$ - and WT-series holes, place large uncertainties on the estimates of the top of the zeolitized horizon (generally at least $\pm 10 \mathrm{~m}$ ). The contact between vitric and zeolitic rocks is also gradational (Levy 1991), adding a further uncertainty of $\sim 10 \mathrm{~m}$ in the location of the zeolitization front.

Given these caveats, there remains a significant number of drill cores with zeolitization horizons about 110-130 $\mathrm{m}$ above the present SWL (Fig. 6). Unfortunately, all of these cores are closely clustered in one central area and are thus of unknown regional significance. The search for ancient SWLs will require the examination of multiple lines of evidence. Combined studies of structural indications of fault displacements of the zeolitization horizon, of glass preservation, and of other mineral distributions (e.g., tridymite) have been interpreted to indicate that rises in the SWL since the zeolitized horizons developed have been no greater than $\sim 60 \mathrm{~m}$ (Levy 1991). Studies of Sr-isotopes in calcite at Yucca Mountain suggest a possible ancient SWL $\sim 85 \mathrm{~m}$ above the present SWL (Marshall et al. 1993). Hydrologic modeling suggests a maximum SWL rise of $130 \mathrm{~m}$ (Czarnecki 1985). At present, it appears that limits on SWL fluctuation may be obtainable even if absolute determinations and timings are not. Levy (1991) points out that the localities with almost no drill-core data, between the eastern margin of the exploration block and the Yucca Mountain crest, are critical to this question, for it is here that the vitric-to-zeolitic transition of the Calico Hills Formation occurs. 


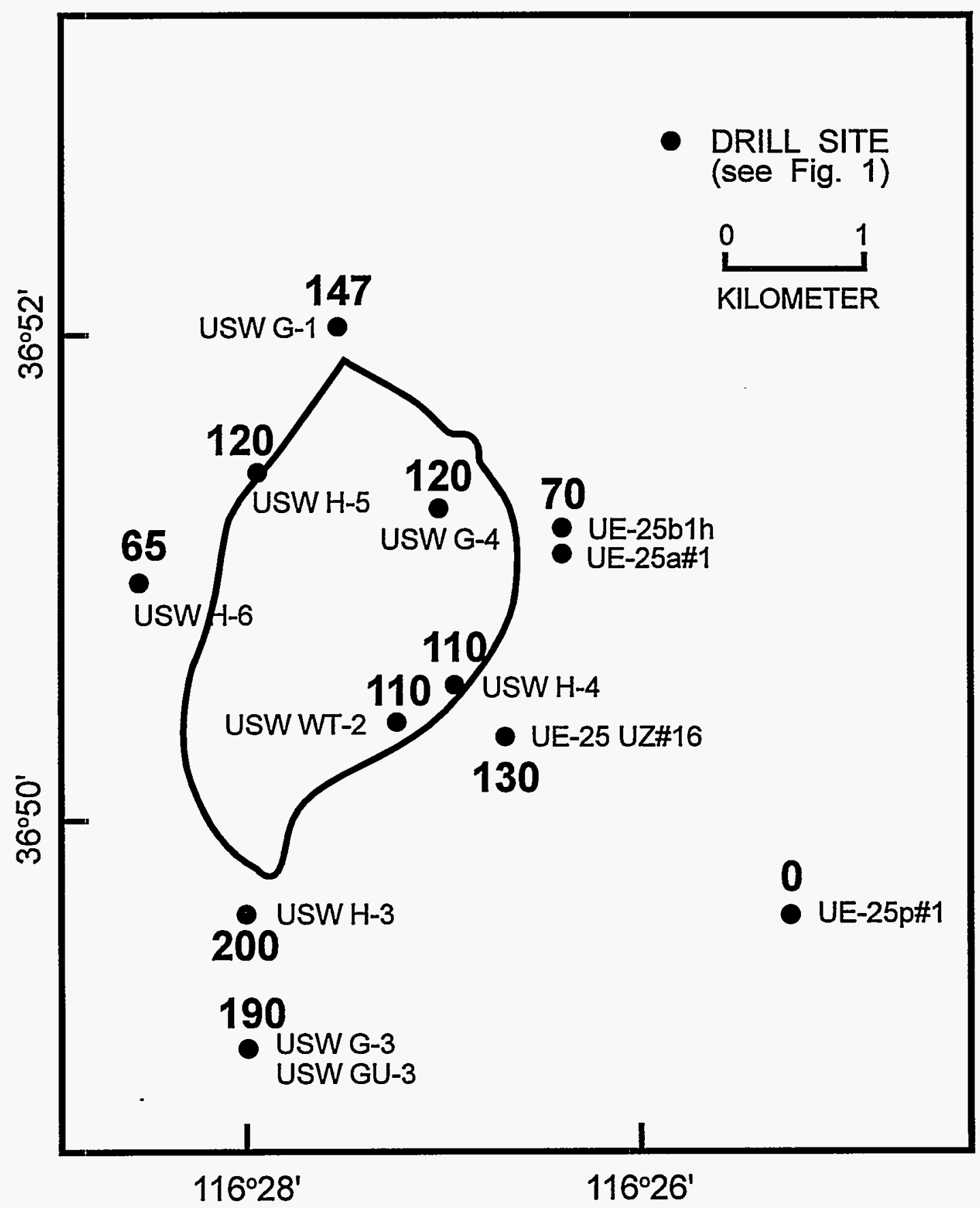

Fig. 6. Elevations above present SWL of the top of the most abundantly zeolitized horizon in drill cores at Yucca Mountain. Inferences of paleo-water table from such data are presently limited by several factors (see Levy 1991) but may be advanced by further studies of samples from cored holes at the Yucca Mountain site. 
Future drilling in this region should provide an important test of the various potential indicators of ancient SWLS.

\section{CONCLUSIONS}

Drill core UE-25 UZ\#16 provides an important addition to previous core determinations of mineral distributions at Yucca Mountain. Previous concepts of mineral distributions (Bish and Chipera 1989) have largely been borne out by the results from UZ\#16. However, closer sample spacing has provided a more exact determination of mineral stratigraphy, particularly in hydrologically important units such as the nonwelded zone above the Topopah Spring Tuff and in the upper vitrophyre of the Topopah Spring Tuff. Moreover, the occurrence of matrix stellerite in the lower half of the devitrified Topopah Spring Tuff of UZ\#16 has shown that, at our present state of knowledge, some unexpected mineralogic features can still be encountered in the exploration of Yucca Mountain by drilling.

\section{ACKNOWLEDGMENTS}

We are grateful to David Broxton and Schön Levy for their reviews of this manuscript. This work was supported by the Yucca Mountain Site Characterization Project Office as part of the Civilian Radioactive Waste Management Program. This Project is managed by the U.S. Department of Energy, Yucca Mountain Site Characterization Project. The Yucca Mountain Project Technical Catalog Data Tracking Number for this work is LA000000000086.002. The record package containing traceability information is LA-EES-1-03-94-003.

\section{REFERENCES}

Alietti, A. (1972) Polymorphism and Crystal-Chemistry of Heulandites and Clinoptilolites, American Mineralogist 57, 1448-1462. NNA.940428.0054

Bish, D. L., and S. J. Chipera (1986) Mineralogy of Drill Holes J-13, UE-25A\#1, and USW G-1 at Yucca Mountain, Nevada, Los Alamos National Laboratory report LA-10764-MS. HQS.880517.1957

Bish, D. L., and S. J. Chipera (1988) Problems and Solutions in Quantitative Analysis of Complex Mixtures by X-Ray Powder Diffraction, in Advances in X-Ray Analysis, C. S. Barrett, J. V. Gilfrich, R. Jenkins, J. C. Russ, J. W. Richardson, Jr., and P. K. Predecki, Eds. (Plenum Press, New York) Vol. 31, 295-308. NNA.890405.0178

Bish, D. L., and S. J. Chipera (1989) Revised Mineralogic Summary of Yucca Mountain, Nevada, Los Alamos National Laboratory report LA-11497-MS. NNA.891019.0029 
Bish, D. L., and S. J. Chipera (1991) Detection of Trace Amounts of Erionite Using X-ray Powder Diffraction: Erionite in Tuffs of Yucca Mountain Nevada, and Central Turkey, Clays and Clay Minerals 39, 437-445. NNA.920319.0003

Bish, D. L., A. E. Ogard, D. T. Vaniman, and L. Benson (1984) Mineralogy-Petrology and Groundwater Geochemistry of Yucca Mountain Tuffs, in Scientific Basis for Nuclear Waste Management VII, Materials Research Society Symposia Proceedings, Boston, Massachusetts (G.L. McVay, Ed.), 283-291. NNA.870406.0085

Bish, D. L., and R. C. Reynolds, Jr. (1989) Sample Preparation for X-ray Diffraction, in Modern Powder Diffraction, D. L. Bish and J. E. Post, Eds. (Mineralogical Society of America, Washington, D.C.) Vol. 20, 73-99. Readily available.

Broxton, D. E., R. G. Warren, F. M. Byers, and R. B. Scott (1989) Chemical and Mineralogic Trends Within the Timber Mountain-Oasis Valley Caldera Complex, Nevada: Evidence for Multiple Cycles of Chemical Evolution in a Long-Lived Silicic Magma System, Journal of Geophysical Research 94, 5961-5985.

Carlos, B. A., S. J. Chipera, D. L. Bish, and R. Raymond (in press) Distribution and Chemistry of Fracture-Lining Zeolites at Yucca Mountain, Nevada, Proceedings of the $4^{\text {th }}$ International Conference of the Occurrence, Properties, and Utilization of Natural Zeolites, Boise, Idaho.

Chipera, S. J., and D. L. Bish (in press) Multi-Reflection RIR and Intensity Normalization for Quantitative Analyses: Applications to Feldspars and Zeolites, Powder Diffraction.

Chipera, S. J., D. L. Bish, and B. A. Carlos (in press) Equilibrium Modeling of the Formation of Zeolites in Fractures at Yucca Mountain, Nevada, Proceedings of the $4^{\text {th }}$ International Conference of the Occurrence, Properties, and Utilization of Natural Zeolites, Boise, Idaho.

Chung, F. H. (1974) Quantitative Interpretation of X-Ray Diffraction Patterns of Mixtures. I. Matrix-Flushing Method for Quantitative Multicomponent Analysis, Journal of Applied Crystallography 7, 519-525. NNA.890405.0179

Czarnecki, J. B. (1985) Simulated Effects of Increased Recharge on the Ground-Water Flow System of Yucca Mountain and Vicinity, Nevada-California, U. S. Geol. Survey Water. Resources Invest. Report. 84-4344. NNA.870407.0008

Ervin, E. M., R. R. Luckey, and D. J. Burkhardt (1993) Summary of Revised PotentiometricSurface Map for Yucca Mountain and Vicinity, Nevada, In High Level Radioactive Waste Management, Amer. Nuc. Soc. and Amer. Soc. Civil Eng., Proc. $4^{\text {th }}$ Ann. Internat. Conf., Las Vegas, Nevada, 1554-1558. NNA.931129.0086

Flint, A. L., L. E. Flint, and J. A. Hevesi (1993) The Influence of Long Term Climate Change on Net Infiltration at Yucca Mountain, Nevada, In High Level Radioactive Waste Management, Amer. Nuc. Soc. and Amer. Soc. Civil Eng., Proc. $4^{\text {th }}$ Ann. Internat. Conf., Las Vegas, Nevada, 152-159. NNA.930615.0054 
Galli, E., and A. Alberti (1975) The Crystal Structure of Stellerite, Bull. Soc. Fr. Miner. Crist. 98, 11-18. NNA.940428.0052

JCPDS (1986) Mineral Powder Diffraction File Data Book (International Centre for Diffraction Data, Swarthmore, Pennsylvania). Readily available.

Kerrisk, J. F. (1987) Groundwater Chemistry at Yucca Mountain, Nevada, and Vicinity, Los Alamos National Laboratory report LA-10929-MS. NNA.870507.0017

Klug, H. P., and L. E. Alexander (1974) X-ray Diffraction Procedures for Polycrystalline and Amorphous Materials (John Wiley \& Sons, Inc., New York). Readily available.

Levy, S. S. (1991) Mineralogic Alteration History and Paleohydrology at Yucca Mountain, Nevada, In High Level Radioactive Waste Management, Amer. Nuc. Soc. and Amer. Soc. Civil Eng., Proc. $2^{\text {nd }}$ Internat. Conf., Las Vegas, Nevada, 477-485. NNA.920131.0296

Levy, S. S., and J. R. O'Neil (1989) Moderate-Temperature Zeolitic Alteration in a Cooling Pyroclastic Deposit, Chemical Geology 76, 321-326. NNA.900820.0270

Lindsley, D. H. (1976) The Crystal Chemistry and Structure of Oxide Minerals as Exemplified by the Fe-Ti Oxides, in Oxide Minerals, D. Rumble, III, Ed. (Mineralogical Society of America, Washington, D.C.) Vol. 3, L1-L60. Readily available.

Lipman, P. W. (1971) Iron-Titanium Oxide Phenocrysts in Compositionally Zoned Ash-Flow Sheets from Southern Nevada, Journal of Geology 79, 438-456. NNA.940428.0053

Marshall, B. D., Z. E. Peterman, and J. S. Stuckless (1993) Strontium Isotopic Evidence for a Higher Water Table at Yucca Mountain, In High Level Radioactive Waste Management, Amer. Nuc. Soc. and Amer. Soc. Civil Eng., Proc. $4^{\text {th }}$ Ann. Internat. Conf., Las Vegas, Nevada, 1948-1952. NNA.931025.0041

Moore, L. M., S. J. Chipera, and D. L. Bish (1993) Estimates of Standard Error Associated with Quantitative XRD Analyses of Phase Abundance, TWS-EES-1-09-93-010, attachment to the Yucca Mountain Site Characterization Project Monthly Activity Report (August/September 1993). NNA.940428.0051

Mumpton, F. A. (1960) Clinoptilolite Redefined, American Mineralogist 45, 351-369. NNA.930629.0012

Post, J. E., and D. L. Bish (1989) Rietveld Refinement of Crystal Structures using Powder Xray Diffraction Data, in Modern Powder Diffraction, D. L. Bish and J. E. Post, Eds. (Mineralogical Society of America, Washington, D.C.) Vol. 20, 277-308. Readily available.

Reynolds, R. C., Jr. and R. C. Reynolds III (1987) Description of Program NEWMOD for the Calculation of the One-Dimensional X-Ray Diffraction Patterns of Mixed-Layered Clays; FORTRAN 77 Version NEWMOD 1.01, R.C. Reynolds, Jr., 8 Brook Dr., Hanover, New Hampshire. NNA.940428.0049 
Smith, D. K., M. C. Nichols, and M. E. Zolensky (1982) POWD10, A FORTRAN IV Program for Calculating X-Ray Powder Diffraction Patterns - Version 10, Pennsylvania State University, College of Earth and Mineral Sciences report. NNA.890602.0030

Torresan, M. (1987) The Use of Sodium Polytungstate in Heavy Mineral Separations, U.S. Geological Survey Open-File Report 87-590. NNA.940428.0048

Vaniman, D. T. (1993) Calcite Deposits in Fractures at Yucca Mountain, Nevada, In High Level Radioactive Waste Management, Amer. Nuc. Soc. and Amer. Soc. Civil Eng., Proc. $4^{\text {th }}$ Ann. Internat. Conf., Las Vegas, Nevada, 1935-1939. NNA.931028.0001

Vaniman, D. T., and D. L. Bish (in press) The Importance of Zeolites in the Potential High-Level Radioactive Waste Repository at Yucca Mountain, Nevada, Proceedings of the $4^{\text {th }}$ International Conference of the Occurrence, Properties, and Utilization of Natural Zeolites, Boise, Idaho.

Vaniman, D., D. Bish, D. Broxton, F. Byers, G. Heiken, B. Carlos, E. Semarge, F. Caporuscio, and R. Gooley (1984) Variations in Authigenic Mineralogy and Sorptive Zeolite Abundance at Yucca Mountain, Nevada, based on Studies of Drill Cores USW GU-3 and G-3, Los Alamos National Laboratory report LA-9707-MS. NNA.870519.0043

Vaniman, D. T., S. J. Chipera, and D. L. Bish (1993) Çombined Quantitative-XRD and Chemical Evaluation of Soil Formation at Yucca Mountain, Nevada, Proceedings of the 1993 Annual Meeting of the Clay Minerals Society, San Diego, California, 55. NNA.940428.0050 


\section{APPENDIX I}

\section{FRACTURE-LINING MINERALS IN DRILL CORE UE-25 UZ\#16}

\section{INTRODUCTION}

Previous studies of fracture coatings in drill cores from Yucca Mountain (Carlos et al. 1993; Carlos et al. in press a; Carlos et al. in press b) have demonstrated that there is a general correlation of fracture-coating mineralogy with stratigraphy, and that coatings in the Topopah Spring Tuff are the most variable with depth and laterally across Yucca Mountain. Thus, fracture coatings in drill core UE-25 UZ\#16 were examined to determine if they followed the patterns of distribution observed in other drill cores at Yucca Mountain that were obtained before implementation of the current QA program.

\section{METHODS}

Fractures throughout UE-25 UZ\#16 were examined visually at up to 50x magnification, with representative samples selected and coatings scraped for analysis by $X$-ray powder diffraction (XRD). Only qualitative XRD analyses of fracture coatings were obtained because of the uneven and often sporadic mineral distributions within a given fracture. The portion of a coating that was scraped may not be quantitatively representative of mineral abundances, either in volume or in percent coverage of the fracture surface, even though the particular mineralogy is qualitatively representative of the fracture. Not all fractures or fracture minerals required XRD analyses, as some coatings were readily identified by visual inspection (e.g., calcite, opal, euhedral quartz, and coarse-grained zeolites).

\section{RESULTS}

The correlation of fracture-coating mineralogy with stratigraphy in drill core UE-25 UZ\#16 appears to extend even to sub-units of the Topopah Spring Tuff. Therefore the following discussion is organized by stratigraphic unit. The stratigraphic breaks are those provided by the United States Geological Survey (USGS) and are available from the Yucca Mountain Technical Data Base (DTN GS931208314211.047). The results of the XRD analyses are given in Table A1 of this appendix and are combined with visual identifications to determine the fracture-lining mineral distributions graphically presented in Figure A1. Figure A1 also provides some whole-rock mineralogy as determined by XRD analysis and a generalized stratigraphy of the drill core so that the relationships between the rock matrix and fracture coatings can be seen. 


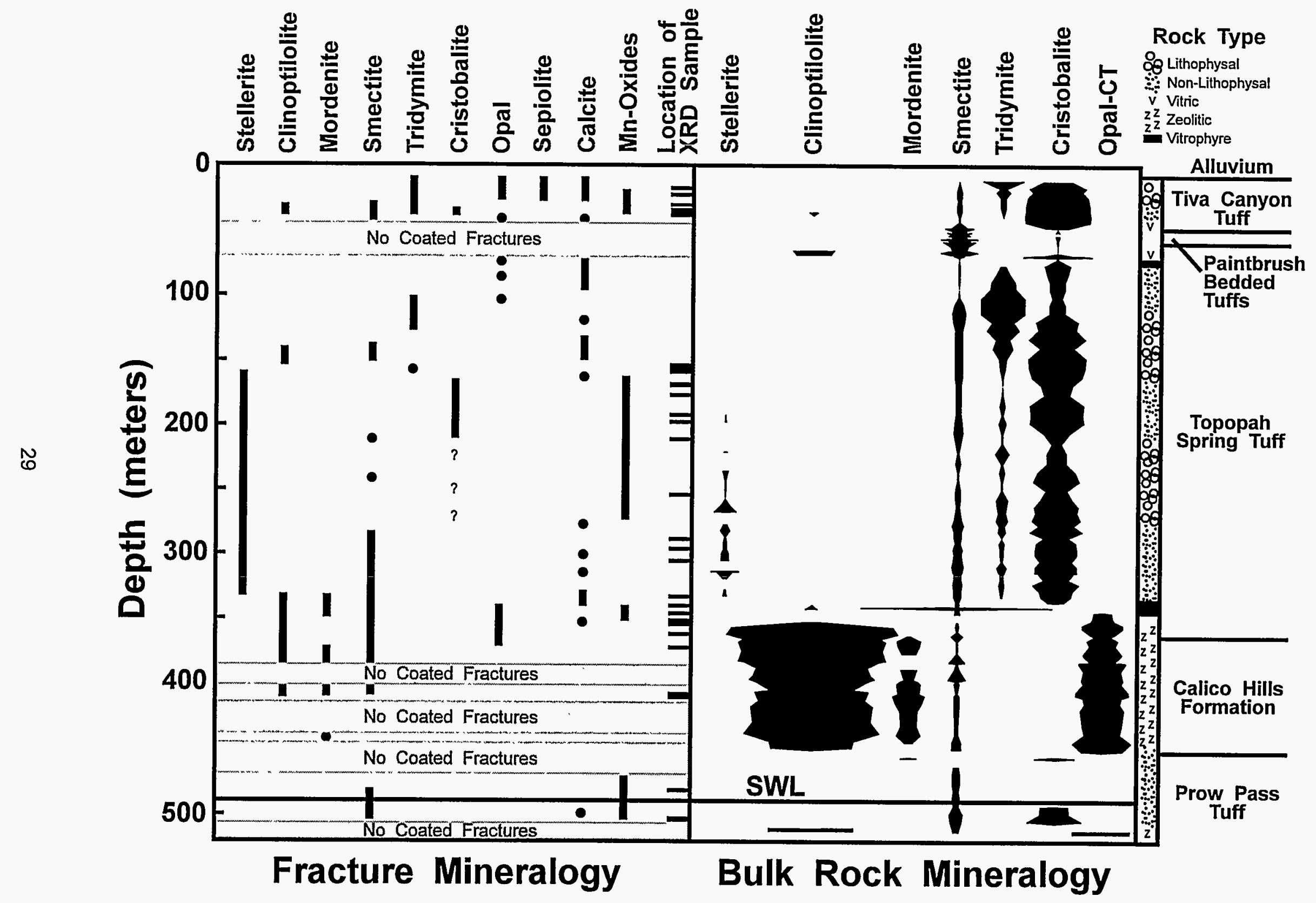

Fig. A1. Distribution of fracture-lining minerals compared with bulk-rock mineralogy in drill core UE-25 UZ\#16. 
Table A1. Qualitative Mineralogy as Determined by XRD for UE-25 UZ\#16 Fracture Samples

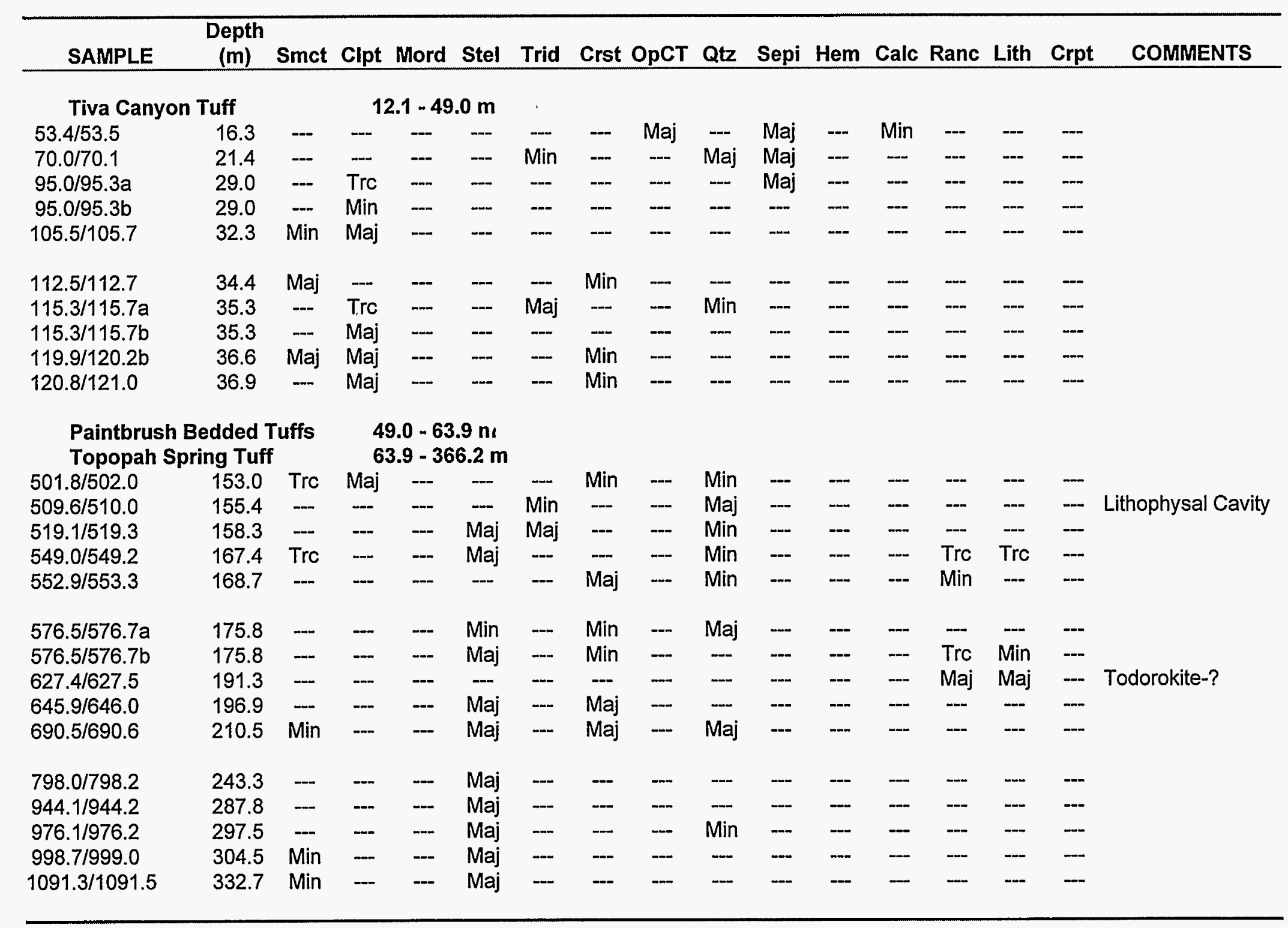


Table A1. Qualitative Mineralogy as Determined by XRD for UE-25 UZ\#16 Fracture Samples (Continued)

\begin{tabular}{|c|c|c|c|c|c|c|c|c|c|c|c|c|c|c|c|c|}
\hline SAMPLE & $\begin{array}{c}\text { Depth } \\
\text { (m) }\end{array}$ & Smct & CIpt & Mord & Stel & Trid & Crst & OpCT & Qtz & Sepi & Hem & Calc & Ranc & Lith & Crpt & COMMENTS \\
\hline $1108.2 / 1108.5$ & 337.9 & -- & Maj & -- & Min? & - & - & - & Min & -- & - & -- & -- & -- & - & \\
\hline $1114.0 / 1114.3$ & 339.6 & Maj & Min & --- & -- & -- & - & Min & -- & - & -- & -- & Min & -- & -- & \\
\hline $1133.3 / 1133.4$ & 345.5 & Maj & Trc & -- & -- & --- & --- & Min & -- & -- & -- & -- & Trc & -- & -- & \\
\hline $1151.8 / 1152.0$ & 351.1 & Maj & Min & $-\cdots$ & -- & -- & -- & Min & -- & -- & -- & Trc & Trc & -- & -- & Trace of Erionite \\
\hline $1160.5 / 1160.7$ & 353.8 & Maj & Min & $-\cdots$ & --- & --- & -- & Min & -- & -- & -- & $--\infty$ & $-\cdots$ & $-\cdots$ & -- & Trace of Erionite \\
\hline $1184.8 / 1185.1$ & 361.2 & $-m$ & Maj & -- & -- & -- & -- & -- & -- & -- & -- & -- & -- & -- & --- & \\
\hline \multicolumn{3}{|c|}{ Calico Hills Formation } & \multicolumn{5}{|c|}{366.2 - 452.6 meters } & & & & & & & & & \\
\hline $1220.4 / 1220.5 a$ & 372.0 & Min & $\operatorname{Min}$ & Maj & $-\cdots$ & -- & -- & Min & $-\infty$ & $-\cdots$ & $-\cdots$ & - & - & - & $-\cdots$ & \\
\hline $1220.4 / 1220.5 b$ & 372.0 & Min & Min & Maj & -- & -- & -- & --- & -- & -- & -- & -- & -- & -- & -- & \\
\hline $1337.0 / 1337.2$ & 407.6 & Maj & Min & Maj & --- & --- & -- & -- & -- & -- & -- & --- & --- & --- & -- & \\
\hline $1346.1 / 1346.2$ & 410.3 & Maj & Min & Min & - & $m$ & -- & $\cdots$ & - & -+ & $\cdots$ & -- & --- & --- & -- & \\
\hline \multicolumn{3}{|c|}{$\begin{array}{l}\text { Prow Pass Member } \\
\text { Static Water Level }\end{array}$} & \multicolumn{5}{|c|}{$\begin{array}{l}452.5 \text { meters to bottom } \\
489.2 \text { meters }\end{array}$} & & & & & & & & & \\
\hline $1649.7 / 1649.9$ & 502.9 & Min & --- & --- & --- & -- & - & -- & Maj & - & Tre & -- & -- & Min & Min & \\
\hline $1653.9 / 1654.2$ & 504.2 & Min & -- & --- & $-m$ & $-\cdots$ & $-m$ & $-m$ & Maj & -- & -- & - & -- & Min & Maj & \\
\hline
\end{tabular}

\section{Key}

\section{Sample numbers are depths in feet.}

Smct $=$ Smectite

Clpt $=$ Clinoptilolite/heulandite

Mord $=$ Mordenite

Stel $=$ Stellerite

Trid $=$ Tridymite

Crst $=$ Cristobalite

OpCT = Opal-CT
Qtz $=$ Quartz

Sepi $=$ Sepiolite

Hem $=$ Hematite

Calc $=$ Calcite

Ranc $=$ Ranceite

Lith $=$ Lithophorite

Crpt $=$ Cryptomelane/hollandite
Maj $=$ Major abundance $-->\approx 20$ wt. $\%$ of the minerals

Min $=$ Minor-abundance $-\approx 5-20 w t . \%$ of the minerals

Trc $=$ Trace abundance $-<\approx 5$ wt. $\%$ of the minerals

--. = Not detected

? = Presence uncertain 


\section{Tiva Canyon Tuff}

UE-25 UZ\#16 intersected the Tiva Canyon Tuff at 12-m (39.7 ft) depth in a zone of flattened lithophysae. Calcite and opal are the most abundant coatings on fractures in the core throughout the Tiva Canyon Tuff in this drill hole. Tridymite coats lithophysal cavities and related fractures in the devitrified Tiva Canyon Tuff (to $43 \mathrm{~m}$ ). White- to cream-colored sepiolite occurs in a few fractures at less than 30-m depth. At depths $>30 \mathrm{~m}$, smectite is the predominant clay, although neither sepiolite nor smectite is widespread or abundant in fractures in this unit. Mn-oxides occur as spots and dendrites from about 19-m depth (in the lithophysal zone) to about $37 \mathrm{~m}$ in the lower part of the non-lithophysal interval, and they range in abundance from $<1 \%$ coverage of a fracture surface at the upper and lower depth limits to as much as $15 \%$ coverage in the middle of the non-lithophysal interval. None of the scraped coatings in this interval contain sufficient amounts of Mn-oxide minerals to be detected by XRD analysis, but Mn-oxide coatings from other Tiva Canyon Tuff fractures at Yucca Mountain are composed of rancieite and lithiophorite (Carlos et al. 1993). From about 28-m depth (in the non-lithophysal interval) to the top of the vitric Tiva Canyon Tuff at about 43-m depth, fracture coatings of small prismatic heulandite crystals increase in frequency and in abundance from sparse $(\sim 2 \%)$ to $100 \%$ coverage. This mineral is presumed to be heulandite, rather than isostructural clinoptilolite, based on the results of heating tests performed on matrix heulandite in the top of the Topopah Spring Tuff as described in the main portion of this report. Calcite is the most abundant fracture coating in the vitric Tiva Canyon Tuff, from about 43- to 47-m depth, with minor amounts of opal and clay (which visually appears to be smectite, but was not analyzed by XRD). Nonwelded tuffs support few fractures, and the bedded and nonwelded tuffs between the Tiva Canyon Tuff and the upper vitrophyre of the Topopah Spring Tuff (about 49- to 70-m depth) contain no coated fractures.

\section{Topopah Spring Tuff}

Calcite and opal are the most abundant fracture coatings in the upper vitrophyre and devitrified upper non-lithophysal intervals of the Topopah Spring Tuff. Calcite and opal may occur alone or together in fractures. When both are present, opal overlies calcite. In the upper non-lithophysal zone (below about $73 \mathrm{~m}$ ), small, clear vapor-phase crystals of tridymite become increasingly abundant.

In the upper lithophysal zone (from about 109 to $167 \mathrm{~m}$ ) tridymite crystals line lithophysal cavities. The gray appearance of tridymite in hand sample is probably caused by very small hematite blades intergrown with tridymite and in the matrix just underlying it. Calcite commonly overlies tridymite in lithophysal cavities and fractures throughout this interval. Euhedral quartz, although present, is not common in the lithophysal cavities and fractures. 
Below about $143 \mathrm{~m}$, some fractures have a $40-100 \%$ coating of small prismatic zeolites. Coatings examined at 143 and $153 \mathrm{~m}$ are heulandite, but below $158 \mathrm{~m}$ the prismatic crystals in fractures are stellerite. This change in zeolite mineralogy occurs about $10 \mathrm{~m}$ above the lithophysal/non-lithophysal transition in the tuff and about $33 \mathrm{~m}$ above the highest occurrence of stellerite in the matrix (Fig. A1). The appearance of stellerite in the fractures does not coincide with any other change in fracture-coating mineralogy. Small crusts of fine-grained white material coat $5-20 \%$ of a few fracture surfaces in this interval. Small crusts covering $<10 \%$ of a fracture surface near the top of the upper lithophysal interval (at $110 \mathrm{~m}$ ) are banded and slightly blue-white in color, resembling mordenite, but there is insufficient coating on the surface to scrape for XRD identification. Mordenite has not been identified in XRD analyses of any fracture coatings in the devitrified Topopah Spring Tuff in this drill core, unlike previous cores drilled at Yucca Mountain (e.g., USW G-1, G-2, GU-3, G-4 and UE-25a\#1, Carlos et al. in press b). The white crusts occurring with heulandite in this interval, and with stellerite just below this lithophysal interval, were identified as smectite in XRD analyses. Therefore the fine-grained white crusts at $110 \mathrm{~m}$ may be smectite and/or cristobalite rather than mordenite.

In the middle non-lithophysal zone (from about 167- to 210-m depth), prismatic stellerite and spots and dendrites of lithiophorite and rancieite are the most abundant fracture coatings. Stellerite covers from 20 to $100 \%$ of individual fracture surfaces, and Mn-oxides coat from 5 to $80 \%$ of the surface on fractures in which they occur. Fine-grained white coatings covering $<5 \%$ of the surface on a few fractures are smectite and/or cristobalite (determined by XRD analysis). "Lithophysal" fractures, so called because they contain silica with tridymite morphology and have bleached margins, cut the core at about 170,180, and 196-199 m. Prismatic stellerite is visible over the tridymite on some open fractures.

In the lower lithophysal interval (about 210-279 m), stellerite continues to be a common and abundant coating. Mn-oxide minerals are present above $243 \mathrm{~m}$ and below $276 \mathrm{~m}$ but are not on all fractures in those intervals and cover less than $5 \%$ of any fracture surface. Silica with tridymite morphology (referred to as "tridymite," which may include other silica phases replacing tridymite) coats "lithophysal" fractures and lithophysal cavities. None of the "tridymite" in this interval was analyzed by $X R D$, but results of the whole-rock XRD analyses for this interval suggest that the silica mineral present is more likely to be cristobalite and/or quartz than tridymite. Prismatic stellerite overlies "tridymite" on some lithophysal surfaces, and it cements shattered zones at about 260 to $261 \mathrm{~m}$ and at $275 \mathrm{~m}$. Fine-grained white crusts that occur with stellerite in the upper portion of the interval (210-240 m) are cristobalite and/or smectite.

In the lower non-lithophysal interval (about 279-338 m), stellerite continues to be the dominant fracture coating. A few fractures have sparse $(<5 \%)$ Mn-oxide spots, mostly underlying stellerite. Calcite occurs over stellerite on fractures at about 281, 299, and $326 \mathrm{~m}$. 
Calcite also overlies "tridymite" at 281 and $310 \mathrm{~m}$ and occurs alone at $322 \mathrm{~m}$. Euhedral quartz crystals occur on lithophysal coatings and with stellerite between $317 \mathrm{~m}$ and the top of the altered interval (334 $\mathrm{m}$ ) just above the basal vitrophyre. A fine-grained white coating, probably smectite and/or cristobalite, occurs in some fractures with and overlying the stellerite (stellerite covers $\sim 100 \%$ of the surface and the fine-grained white material covers $5-20 \%$ of the stellerite). The interval immediately above the basal vitrophyre (337-338 m) contains increasing amounts of orange alteration in the matrix and heulandite (visual identification based on crystal morphology) in the fractures. Some fractures also contain calcite or opal. Larger tabular heulandite, common in this interval in other drill cores, is not present in UE-25 UZ\#16.

Fractures in the vitrophyre contain fine-grained white to blue-white coatings with desiccation cracks and Mn-oxide (rancieite) dendrites. XRD analysis of samples from this interval identified the white coatings as a mixture of smectite, opal-CT, and clinoptilolite, although no crystals were visible. Fractures at 351.1 and $353.8 \mathrm{~m}$ also contain small clear acicular crystals that were identified by XRD analysis as erionite, although erionite is only a trace component of the fracture coatings, which are predominantly smectite. Calcite cements breccia in a fracture at $352 \mathrm{~m}$. The few coated fractures in the partially devitrified Topopah Spring Tuff below the basal vitrophyre contain clinoptilolite and calcite.

\section{Calico Hills Formation}

As in other drill cores, mordenite is the dominant fracture coating in the Calico Hills Formation, although clinoptilolite is the dominant mineral in the tuff matrix. The thickness of coatings varies from thin and discontinuous to thicker satiny or ropy coatings in which the fibrous nature of the mordenite is clearly visible. XRD analyses also identified smectite and clinoptilolite in these coatings. Opal-CT was identified in a fracture at $372 \mathrm{~m}$ and is probably present in many of the fractures that were not analyzed by XRD. Planar fractures at 372, 379, and $380 \mathrm{~m}$ have a red-brown stain that probably contains hematite and possibly quartz. Mnoxides appear to be present on the slickensided fracture at $372 \mathrm{~m}$. Intervals from 383 to $402 \mathrm{~m}$, from 412 to $444 \mathrm{~m}$, and below $445 \mathrm{~m}$ into the top of the Prow Pass Tuff have no coated fractures; the few fractures that were not explicitly marked as man-made are probably drillingand handling-induced rather than natural.

\section{Prow Pass Tuff}

No coated fractures were found in the non-welded top (452-471 $\mathrm{m}$ ) and bottom (504$514 \mathrm{~m}$ ) of the Prow Pass Tuff in this drill hole. Fractures are not abundant throughout the middle portion of the Prow Pass Tuff (471-504 m), and almost all contain Mn-oxides, some with red-brown hematite. In the partially welded interval above about $486 \mathrm{~m}$, the Mn-oxides often 
appear to be diffused into the matrix adjacent to hairline fractures (mostly closed) which are traceable only by black discoloration and small air gaps. A fracture at $488-490 \mathrm{~m}$ in the moderately welded interval contains Mn-oxides, hematite, and white crystals that may have originally been vapor-phase tridymite but are now probably quartz. Just below the depth where water was first observed in the hole $(490 \mathrm{~m})$, much of the core had already been removed from the box as natural-state samples and was not available for examination. Fractures on core remaining in the box within the next four meters had no coatings. A calcite-coated vertical fracture was intersected by the edge of the core at $500 \mathrm{~m}$. The remaining few fractures from core below the water table contained the following minerals: Mn-oxides that were identified by XRD as cryptomelane/hollandite and lithiophorite; quartz, either euhedral under Mn-oxide minerals or as fine-grained quartz intergrown with Mn-oxides; hematite; and, on at least one fracture $(504 \mathrm{~m})$, mordenite over cryptomelane, although mordenite was identified visually and not by XRD.

\section{DISCUSSION}

The fracture coatings in this drill core are similar in many respects to those in other drill cores. For example, the variety of fracture coatings in the Paintbrush Group is greater than in deeper tuffs. Sepiolite and heulandite have also been identified from the Tiva Canyon Tuff in drill hole USW GU-3, although not in USW G-4, the only other drill cores in which Tiva Canyon Tuff fractures have been examined. As in other cores, the dominant fracture coating in the zeolitic Calico Hills Formation is mordenite although the most abundant zeolite in the matrix is clinoptilolite. Hematite and Mn-oxides (cryptomelane/hollandite and lithiophorite) are the most common coatings in the devitrified Prow Pass Tuff, with lesser amounts of calcite and quartz. Fracture coatings in the Prow Pass Tuff in UE-25 UZ\#16 are not as abundant as in some other drill cores, but their rarity may be primarily a function of the paucity of fractures (because of the lesser degree of welding).

The most significant differences between the fracture coatings from UE-25 UZ\#16 and those from other drill cores from Yucca Mountain occur in the Topopah Spring Tuff. The very thick interval in the Topopah Spring Tuff (143- to 334-m depth) throughout which stellerite is the principal fracture coating is greater than has been seen in any other drill core. Most of this interval coincides with the occurrence of stellerite in the matrix, although fracture-lining stellerite occurs at shallower depths in the drill hole than matrix stellerite. Matrix stellerite has not yet been identified in any other drill core at Yucca Mountain. As suggested in the main section of this report, the occurrence of matrix stellerite might be related to the location of this drill hole within the imbricate fault zone. There are several intervals of shattered or brecciated rock, generally cemented with stellerite, between $234-$ and $321-\mathrm{m}$ depth, and most of the stellerite in 
the matrix occurs over this intermittently shattered or brecciated interval. In a few instances, the stellerite content of the matrix increases greatly in the vicinity of one of these shattered zones, but not all shattered zones show an increase in matrix stellerite. Many of the brecciated intervals are associated with lithophysal cavities, and stellerite cements fragments of "tridymite" and vapor-phase altered matrix. Quartz crystals at $317 \mathrm{~m}$ overlie stellerite, and at $318 \mathrm{~m}$ they appear to be intergrown with stellerite. None of the fracture-coating phases has been dated, but mineral-intergrowth and rock-fracture relationships suggest that stellerite deposition may have occurred early in the history of the mountain.

The lack of heulandite in fractures in the lower Topopah Spring Tuff is also unusual since heulandite is a more common fracture coating in most drill cores than stellerite, and the two coexist in some drill cores (UE-25a\#1, USW G-1, USW G-2). There appears to be a change from heulandite as the prismatic zeolite in fractures at the top of the Topopah Spring Tuff in UE-25 UZ\#16 to stellerite, and then, just a meter above the basal vitrophyre, heulandite occurs again in the fractures. If at least some of the heulandite in other drill cores were deposited more recently than most fracture-lining minerals, as suggested by Carlos et al. (in press $a, b)$, then the distribution of heulandite in UE-25 UZ\#16 suggests that the most recent fracture flow at this location was limited to the intervals at the top of the drill hole (above 143 m). Levy and O'Neil (1989) postulated that zeolites just above the basal vitrophyre were formed during early devitrification of the tuff. The presence of two morphologies and two or more compositions of heulandite in the same fractures in other drill cores (UE-25a\#1, USW G1, USW G-2, USW G-4) suggests the occurrence of more than one period of deposition. In a few fractures (e.g., at $357 \mathrm{~m}$ in UE-25a\#1) the presence of heulandite both under and over calcite clearly indicates the existence of two intervals of heulandite deposition. The small $(<5-$ $\mu \mathrm{m}$ long) size of the prismatic crystals over calcite does not permit chemical analyses for comparison with the tabular crystals that are clearly under calcite, or with the larger $(40-50 \mu \mathrm{m})$ prismatic crystals that are in the same fractures but not in contact with calcite. In some fractures tabular crystals appear to be zoned (Carlos et al. in press), which may be the result of more than one episode of zeolite deposition or may reflect a change in conditions during a single episode. The distribution of large tabular heulandite crystals in fractures in other drill cores appears to correlate with a zeolitic interval of tuff immediately above the vitrophyre. Tabular heulandite is absent in fractures from UE-25 UZ\#16 as it is in USW GU-3. If heulandite is present in the matrix above the basal vitrophyre of the Topopah Spring Tuff in UE-25 UZ\#16, it must be within the unanalyzed 2-m spacing between the last sample in the devitrified tuff and the top of the vitrophyre as none was detected in XRD analyses. Data need to be collected from additional drill cores before we can interpret the significance of the transition from stellerite 
to heulandite and the absence of tabular heulandite in fractures in the lower devitrified Topopah Spring Tuff in UE-25 UZ\#16.

Another difference between the fractures in the Topopah Spring Tuff in UE-25 UZ\#16 and those in other drill cores is the near absence of mordenite in UE-25 UZ\#16. Fine-grained mordenite is an early fracture coating in the devitrified Topopah Spring Tuff in most of the drill holes at Yucca Mountain. Coarser mordenite commonly accompanies heulandite in the fractures and is interpreted as having been deposited later than the fine-grained mordenite. Fine-grained mordenite is almost entirely absent from fractures in UE-25 UZ\#16. Examination of future drill cores may help establish a pattern to the mordenite distribution and refine our interpretation of the timing and conditions of deposition of zeolites in fractures of the Paintbrush Group.

Other fracture-lining minerals in the Paintbrush Group are similar to those in other drill holes at Yucca Mountain. Rancieite and lithiophorite are the dominant Mn-oxide minerals in the Paintbrush Group. The coatings in the basal vitrophyre and underlying partially welded Topopah Spring Tuff are also similar to those in other drill cores and include smectite, clinoptilolite, opal-CT, calcite, rancieite, and possibly mordenite.

\section{CONCLUSIONS}

The fracture coatings in all intervals except the devitrified Topopah Spring Tuff in UE-25 UZ\#16 resemble those of previously examined drill cores from Yucca Mountain. The presence of heulandite in fractures in the lower non-lithophysal Topopah Spring Tuff in UE-25 UZ\#16, as in USW GU-3, is limited to an interval about a meter thick immediately above the lower vitrophyre; however, tabular heulandite is absent. The extensive interval containing fracturelining stellerite and the near absence of mordenite in fractures of the Topopah Spring Tuff in UE-25 UZ\#16, however, are unique among all the core examined to date. $A$ better understanding of the factors controlling fracture-lining zeolites is needed before we can infer fracture-flow history in the unsaturated zone from fracture fillings.

\section{ACKNOWLEDGMENTS}

This work was supported and managed by the U.S. Department of Energy, Yucca Mountain Site Characterization Office. The Los Alamos data tracking number for this appendix is LA-000000000100.001. The record package containing traceability information is LA-EES-1TIP-94-007. 


\section{REFERENCES}

Carlos, B. A., S. J. Chipera, and D. L. Bish (in press, a) Distribution and Chemistry of Fracturelining Minerals at Yucca Mountain, Nevada, Los Alamos National Laboratory report.

Carlos, B. A., S. J. Chipera, D. L. Bish and R. Raymond (in press, b) Distribution and Chemistry of Fracture-lining Zeolites at Yucca Mountain, Nevada, Proceedings of the $4^{\text {th }}$ International Conference on the Occurrence, Properties, and Utilization of Natural Zeolites, Boise, Idaho.

Carlos, B. A., S. J. Chipera, D. L. Bish, and S. J. Craven (1993) Fracture-lining Manganese Oxide Minerals in Silicic Tuff, Yucca Mountain, Nevada, U.S.A., Chemical Geology 107, 47-69. NNA.931028.0002

Levy, S. S., and J. R. O'Neil (1989) Moderate-Temperature Zeolitic Alteration in a Cooling Pyroclastic Deposit, Chemical Geology 76, 321-326. NNA.900820.0270 


\section{APPENDIX \|}

\section{QUALITY ASSURANCE}

The following is a list of YMP logbooks and notebooks containing data presented in this report.

MAIN REPORT

APPENDIX I
LOG/NOTEBOOK

TWS-EES-1-3-89-17

TWS-ESS-1-8/86-57

TWS-ESS-1-7/86-35

TWS-EES-1-1-92-03

LOG/NOTEBOOK

TWS-EES-1-3-89-17

TWS-ESS-1-1-90-1

TWS-ESS-1-7-91-10

TWS-EES-1-1-93-4

TWS-EES-1-1-93-5

TWS-EES-1-3-93-8

\section{PAGES}

$33-43$

$38-43$

55-59

$52-53$

\section{PAGES}

48,50

120-123

43-60, 69-75

61-62, 93-119, 145-147

2-14, 43-63

54

A list of the raw-data computer files is included as part of the records package for this report.

\section{COMPUTER SOFTWARE}

Data were obtained on the LANL EES-1 Siemens D500 X-ray powder diffractometers using the commercial software package-DIFFRAC5000.

LANL YMP release label $=$ DIFFRAC5000-01-00-00.

Integrated peak intensities were obtained using the program GRAPHINT.

LANL YMP release label $=$ GRAPHINT-01-00-00.

QUANT (version 5.04) had been qualified under the software QA requirements of the earlier QA program which included auxiliary software.

LANL YMP release label = QUANT-01-00-00.

The latest modifications to QUANT (producing version 5.05) were conducted in accordance with the QA program emplaced 31-JAN-1994 and are documented in TWS-ESS-1-1-92-03, pages 54-70. 